\title{
4 Lesartendifferenzierung
}

Die Unterscheidung von Lesarten ,ist eines der heikelsten und meistdiskutierten Probleme der Semantik und Lexikographie“ (Stein 2005: 57). Sie ist jedoch notwendig, will man im Sinne der eingangs formulierten Forschungsfragen F1 und F2 fundierte Aussagen zur Argumentweglassung sowie zu daraus möglicherweise resultierenden semantischen Konsequenzen machen. Die vorangehenden Darstellungen haben gezeigt,

dass das Diktum der älteren Valenztheorie Das Verb bestimmt Zahl und Art der Ergänzungen viel zu kurz greift. [...]

Eine Aussage wie „geben verlangt drei Mitspieler, einen Gebenden, der als Subjekt, einen Empfänger, der als eine Dativergänzung, und etwas Gegebenes, das als Akkusativergänzung realisiert wird“ geht von einer bestimmten Verblesart aus. Man denkt nicht an geben als Existenzverb (Beispiel (7)) und auch nicht an Austauschlesarten wie etwas für. bzw. um etwas geben, für die eine Dativergänzung weniger konstitutiv ist als eine für-bzw. um-Präpositivergänzung (Beispiel (8)):

(7) Im Bahn-Radsport gibt es Zeitfahren über verschiedene Distanzen.

(8) Seele und Leib geben wir für unsere Freiheit.

Es sind also eigentlich Verblesarten, von denen man sagen kann, dass sie Zahl und Art der Ergänzungen bestimmen [...].

(Fischer 2010: 29f.)

Im Folgenden soll der Frage nach der Ermittlung von Verwendungsweisen sprachlicher Ausdrücke (hier: Verben) nachgegangen werden. Um einen Einblick in die lexikographische Praxis zu bekommen, werden drei große (Valenz-)Wörterbücher der Gegenwartssprache betrachtet (Kapitel 4.1). Im Einzelnen handelt es sich hierbei um

- DUDEN - das große Wörterbuch der deutschen Sprache,

- DWDS - das digitale Wörterbuch der deutschen Sprache der berlin-brandenburgischen Akademie der Wissenschaften und

- E-VALBU - das elektronische Valenzwörterbuch deutscher Verben des Instituts der deutschen Sprache Mannheim.

Bei der Beschreibung der Wörterbücher geht es vor allem darum, die Kriterien, die bei der Unterscheidung von Verwendungsweisen sowie deren Zusammenfassung eingesetzt werden, zu explizieren. Es wird sich zeigen, dass

Lesarten nicht in demselben Maße objektiv gegeben [sind, TvdB] wie z. B. Tierarten, sondern sie stellen (auch) Konstruktionen des Semantikers, Syntaktikers und Lexikographen dar. Je genauer man hinschaut, desto komplizierter wird das Bild, unterschiedliche Einteilungen sind möglich.

(Âgel/Fischer 2010: 266) 
So ist es „often not possible to determine exactly how many different meanings a word actually has [...]. Symptomatic of this state of affairs is the fact that dictionaries can differ with respect to the number of senses that they list.“ (Taylor 2003a: 640). Daher werden zusätzliche, die traditionellen Verfahren der Polysemiedifferenzierung einschließende Kriterien zur Lesartenunterscheidung dargestellt (Kapitel 4.2). Berücksichtigt werden die Angabe von Paraphrasen und Synonymen, die Analyse syntaktischer Aspekte, die Kollokations- und Kolligationsanalyse sowie die Analyse rollenspezifischer Forderungen.

Die Darstellungen werden zeigen, dass auch die traditionellen Verfahren zur Polysemiedifferenzierung z. T. mit einer Vielzahl unterschiedlicher Probleme verbunden sind; aus diesem Grund wird zur Überprüfung bestimmter Bedeutungshypothesen zusätzlich ein Fragebogen entwickelt (Kapitel 4.3). Der Fragebogen als „klassisches empirisches Erhebungsinstrument“ (Betz 2016: 99) eignet sich hierfür in besonderem Maße, da „eine gleichzeitige Durchführung mit mehreren Befragten möglich ist und die gleichen Informationen bei allen Personen erhoben werden“ (ebd.) können.

Die folgenden Ausführungen dienen neben einem problemorientierten Überblick über die unterschiedlichen Verfahren zur Lesartendifferenzierung vor allem auch dazu, eine für die in Kapitel 5 sich anschließenden Einzelverbuntersuchungen brauchbare Untersuchungs- und Beschreibungsmethode zu entwickeln, mit deren Hilfe sich die unterschiedlichen Verwendungsweisen der hier zu analysierenden Verben möglichst effizient und nach objektivierbaren Kriterien ermitteln und darstellen lassen.

\subsection{Wörterbuchartikel im Vergleich}

Die Sichtung und Zugrundelegung verschiedener Wörterbucheinträge bei der Lesartendisambiguierung erscheint deshalb zweckmäßig, da Wörterbücher im Kern die unterschiedlichen Bedeutungen und Verwendungen von Wörtern beschreiben. Die einzelnen Lesarten eines Ausdrucks werden in Wörterbuchartikeln durch arabischebzw. römische Ziffern und/oder Klein-bzw. Großbuchstaben - $u$. U. auf mehreren Ebenen - untergliedert. Enger zusammenhängende Verwendungsweisen werden dabei häufig, aber nicht immer, in Gruppen zusammengefasst. Diese, in der gegenwärtigen lexikographischen Praxis übliche Vorgehensweise hat ihren Ursprung bereits im Deutschen Wörterbuch von Jacob und Wilhelm Grimm; im Vorwort zu Band 1 heißt es:

Beim beginn des werks schien noch steif und raumverschwendung, die verschiedenheit der bedeutungen in beigefügten zahlen hervorzuheben, wodurch auch hin und wieder die fugen des zusammenhangs versteckt werden könnten. bald aber stellte sich heraus, dasz 
kein gröszerer artikel solcher zahlen entbehren durfte und dasz auch die kleineren dabei mehr gewönnen als verlören. es ist daher in dieser hinsicht mehr gleichförmigkeit eingetreten, die man nur in den ersten lieferungen zuweilen vermissen wird. (Grimm (1854): XLVI)

Ein Rückgriff auf die in der lexikographischen Praxis vorgenommene Lesartenunterscheidung erscheint dort unproblematisch, wo die einzelnen Wörterbücher ähnliche Verwendungsweisen der zu untersuchenden Verben angeben. Daneben sind jedoch auch zahlreiche Unterschiede zu erkennen: „Dictionaries differ in the number of senses they define for each word, the grouping into sub-senses and the content of definitions“ (Ravin/Leacock 2000: 1). Die „Spannbreite ist gerade bei stark polysemen Wörtern bemerkenswert“ (Kunze/Lemnitzer 2007: 18). Beim Vergleich der Wörterbucheinträge des Verbs spielen in den oben genannten Wörterbüchern zeigt sich z.B., dass die unterschiedlichen Hauptverwendungsweisen zwischen 15 (DUDEN), 8 (DWDS) und 23 (E-VALBU) variieren und dass nicht alle Verwendungsweisen in den Wörterbüchern in gleicher Weise aufgeführt werden $^{88}$. Auch die Zusammenfassung einzelner Lesarten erfolgt - sofern sie überhaupt vorgenommen wird (vgl. die grundsätzlich ausbleibende Gruppierung in E-VALBU) - keineswegs einheitlich.

Die z. T. sehr großen Abweichungen in der lexikographischen Beschreibung sind u. a. darauf zurückzuführen, dass der den Wörterbüchern zugrundeliegende Wortschatz unterschiedlich erfasst wird, die Wörterbücher jeweils eigene, häufig nicht objektivierbare Kriterien zur Abgrenzung von Verwendungsweisen benutzen und die Gruppierungen einzelner Lesarten, sofern sie überhaupt erfolgen, ebenfalls divergieren.

\subsubsection{DUDEN - das große Wörterbuch der deutschen Sprache}

DUDEN - das große Wörterbuch der deutschen Sprache liegt als CD-Rom-Fassung vor (DUDEN 20124). Es handelt sich um ein synchrones Wörterbuch, das ,den aktuellen Wortschatz des modernen Deutsch möglichst umfassend darstellen“ soll (ebd. 1). Basis für die lexikographische Arbeit bildet dabei das 2001 begründete und seitdem kontinuierlich weiterentwickelte DUDEN-Korpus. Darüber hinaus „werden auch die Möglichkeiten genutzt, die das Internet für die Recherche nach Wörtern, Wortformen und Wortbedeutungen bietet.“ (ebd. 3) Hiermit stehen nach Ansicht der Autoren vielfältige Quellen zur Verfügung, aus denen verlässliche

88 Taylor (2003a) macht eine ähnliche Beobachtung für das englische Verb <open>. Während es in Collins English Dictionary mit 13 open-Lesarten gelistet ist, wird es im Longman's Dictionary of Contemporary English mit nur fünf Lesarten erfasst. 
Informationen zum aktuellen Stand der deutschen Sprache gewonnen werden können.

Das Hauptanliegen des Großen Wörterbuchs der deutschen Sprache besteht darin, sprachliche Bedeutungsstrukturen darzustellen und dabei zu einer „möglichst exakten Bestimmung ihrer semantischen Vielfalt“ (ebd.: 6) zu gelangen. Nach welchen Kriterien eine entsprechende Lesartendisambiguierung vorgenommen wird, wird jedoch nicht expliziert. Auch auf Anfrage werden die entsprechenden Kriterien nicht offen gelegt; stattdessen heißt es:

Die Redakteure und Redakteurinnen haben für die aus dem Korpus gewonnenen Kontextbelege eines Wortes die im jeweiligen Kontext zutreffenden Bedeutungsparaphrasen aufgrund ihrer muttersprachlichen Kompetenz ermittelt und die Lesarten voneinander unterschieden, in denen die Bedeutungsparaphrasen unterschiedliche semantische Merkmale erkennen ließen. [...] Eine exakte Grenzziehung nach strikt objektiven Kriterien wird hier kaum möglich sein.

(E-Mail-Kontakt vom 12.09.2013)

Bei genauerer Betrachtung scheint vor allem die Angabe von Paraphrasen bzw. Synonymen von zentraler Bedeutung für die Unterscheidung von Verwendungsweisen zu sein, vgl. (86):

\section{akzeptieren}

i.S.v. annehmen, hinnehmen, billigen; anerkennen; mit jemandem oder etwas einverstanden sein (s. DUDEN 2012 ${ }^{4}$ akzeptieren)

Darüber hinaus, das konnte Bons (2009) in einer Untersuchung zu Adjektiven zeigen, finden auch Angaben zu den Entitäten, auf die sich die Argumentstellen der jeweiligen Lesarten beziehen können - vgl. z. B. die Ausführungen zu hart hb $_{1 b}$ (in Bezug auf Geld) stabil, sicher -, sowie syntaktische Kriterien - vgl. etwa die Lesartenbeschreibung von hart $_{5}$ : <in Verbindung mit Präposition> ganz dicht, nahe - Berücksichtigung. Wie und in welchem Umfang nach den genannten Gesichtspunkten gearbeitet wird, wird jedoch nicht ersichtlich.

Die ermittelten Verwendungsweisen werden im DUDEN zum Teil zu Gruppen zusammengefasst. Wie diese Einteilung erfolgt, wird aber nicht angegeben. Es heißt lediglich: „Bei Wörtern mit mehreren Unterbedeutungen werden sie mittels arabischer Ziffern untergliedert. Wo Unterbedeutungen semantisch eng verwandt sind, wird mithilfe von Kleinbuchstaben unterschieden.“ (DUDEN 2012: 8) Es werden keine Angaben dazu gemacht, mithilfe welcher Kriterien man zu einer „engen semantischen Zusammengehörigkeit“ (ebd.) zwischen den Verwendungsweisen gelangt. Ebenso wenig wird innerhalb der Wörterbucheinträge die Art der semantischen Nähe aufgezeigt. 


\subsubsection{DWDS - das digitale Wörterbuch der deutschen Sprache}

DWDS - das digitale Wörterbuch der deutschen Sprache versteht sich als Wortauskunftssystem zur deutschen Sprache in Geschichte und Gegenwart. Es wurde an der berlin-brandenburgischen Akademie der Wissenschaften entwickelt und verfolgt das Ziel der

Schaffung eines „Digitalen Lexikalischen Systems“ - eines umfassenden, jedem Benutzer über das Internet zugänglichen Wortinformationssystems, das Auskunft über den deutschen Wortschatz in Vergangenheit und Gegenwart gibt.

(Geyken 2011a: 9)

Hierzu wurde mit der Web-Seite https://www.dwds.de/ (Stand: 25.05.2018)

eine Benutzeroberfläche geschaffen, die zum einen als Rechercheumgebung eines lexikografischen Arbeitsplatzes für die wissenschaftliche Analyse des deutschen Wortschatzes fungiert, zum anderen aber jedem Interessierten viele Nachschlagemöglichkeiten eröffnet.

(DWDS; cf. Hintergrund)

Kern des DWDS-Wortinformationssystems sind die digitalen Versionen folgender Wörterbücher: das Wörterbuch der deutschen Gegenwartssprache und seine Überarbeitung im DWDS-Wörterbuch, das Etymologische Wörterbuch des Deutschen von Wolfgang Pfeifer, das Deutsche Wörterbuch von Jacob und Wilhelm Grimm sowie dessen Neubearbeitung nach Fertigstellung der geplanten Digitalisierung. Darüber hinaus sind Teile des Großen Wörterbuchs der deutschen Sprache in 10 Bänden („DUDEN 1999“) enthalten ${ }^{89}$ (vgl. DWDS; cf. Wörterbücher).

Nachfolgende Verweise auf Wörterbucheinträge des DWDS beziehen sich vor allem auf das Wörterbuch der deutschen Gegenwartssprache (WDG). Es ist seit März 2003 auf der DWDS-Webseite unter dem Namen Elektronisches Wörterbuch der deutschen Gegenwartssprache (eWDG) veröffentlicht und wurde in „den Folgejahren [...] schrittweise weiter inhaltlich erschlossen und ausgezeichnet“ (ebd.). Den Darstellungen der Webseite ist nicht zu entnehmen, welche Kriterien bei der Lesartendifferenzierung dort zugrundegelegt werden. Das trifft sowohl auf das ursprüngliche WDG als auch auf die vom DWDS vorgenommenen Anpas-

89 Als weitere Quelle steht der kollaborativ erstellte OpenThesaurus zur Verfügung. Hinzu kommen Textkorpora - im Einzelnen das zeitlich und nach Textsorten ausgewogene DWDSKernkorpus, das historische Referenzkorpus des Deutschen Textarchivs und die vorwiegend aus elektronischen Zeitungsquellen stammenden DWDS-Ergänzungs- und Spezialkorpora - sowie wortbezogene statistische Auswertungen (vgl. DWDS; cf. Textkorpora). „In der Web-Ansicht führt das DWDS-Wortinformationssystem diese Quellen zusammen“ (DWDS; cf. Wörterbücher). 
sungen und Überarbeitungen $\mathrm{zu}^{90}$. Auch auf Nachfrage werden die verwendeten Kriterien nicht offen gelegt; stattdessen heißt es:

Eine ausführlichere Darstellung, nach welchen Verfahren und Regeln wir in der laufenden lexikographischen Praxis Bedeutungen differenzieren, würde den Rahmen einer E-Mail-Antwort leider übersteigen. Aber wir bereiten dazu eine Publikation ${ }^{91}$ auf der DWDS-Webseite vor.

(E-Mail-Kontakt vom 28.04.2015; Fußnote ergänzt, TvdB)

Nach Sichtung zahlreicher Wörterbucheinträge scheint allerdings - ähnlich wie im DUDEN - auch hier die Angabe von Paraphrasen bzw. Synonymen ein zentrales Kriterium für die Lesartenunterscheidung zu sein, vgl. (87). Welche weiteren Unterscheidungsmerkmale herangezogen werden, wird jedoch nicht deutlich.

\section{akzeptieren}

i.S.v. etw. annehmen (s. DWDS_eWDG_akzeptieren; 30.05.2018) ${ }^{92}$

Als Gliederungsaspekt der einzelnen Lesarten in den Wörterbucheinträgen wird u. a. die Häufigkeit im Sprachgebrauch genannt. Ein weiterer Faktor kann nach Aussage der Autoren die „Ursprünglichkeit“ der Bedeutung sein; denn es steht die ursprüngliche Bedeutung vor der übertragenen, die sinnlich-konkrete vor der abstrakten, die gemeinsprachliche vor der fach- oder sondersprachlichen. Welche Regeln tatsächlich zur Anwendung kommen, ist nur im Zusammenhang der besonderen semantischen Gegebenheiten eines spezifischen Wortes zu bestimmen (vgl. E-Mail-Kontakt vom 28.04.2015).

\subsubsection{E-VALBU - elektronisches Valenzwörterbuch}

E-VALBU - das elektronische Valenzwörterbuch deutscher Verben des Instituts für deutsche Sprache Mannheim umfasst - wie der Titel bereits besagt - ausschließlich Verben und kann über die Web-Seite http://hypermedia.ids-mannheim.

90 „Das DWDS hat das WDG digitalisiert, fürs Web aufbereitet und auch inhaltlich schon an vielen Stellen überarbeitet.“ (E-Mail-Kontakt vom 31.07.2014)

91 Auch nach Abschluss der vorliegenden Arbeit liegt eine entsprechende Publikation (noch) nicht vor.

$92<$ DWDS_eWDG_akzeptieren; 30.05.2018> steht hier für die vollständige Angabe <,akzeptieren“, In: Wörterbuch der deutschen Gegenwartssprache (1964-1977), kuratiert und bereitgestellt durch das Digitale Wörterbuch der deutschen Sprache, <https://www.dwds.de/wb/wdg/akzeptieren>, abgerufen am 30.05.2018.) >. Im Folgenden wird nur noch die entsprechende Kurzform (DWDS_eWDG_[Verb; Abrufdatum] verwendet, wenn auf Wörterbucheinträge des eWDG im DWDS verwiesen wird. 
de/evalbu/index.html (Stand: 30.05.2018) abgerufen werden. E-VALBU basiert auf dem gedruckten Valenzwörterbuch VALBU, ist jedoch keine einfache elektronische Kopie des Papierwörterbuchs (vgl. Schumacher et al. 2004). Die Aussagen in VALBU wurden anhand des Deutschen Referenzkorpus (DeReKo) überprüft und wo nötig überarbeitet und ergänzt. Zudem wurden die angegebenen Verwendungsbeispiele aktualisiert.

Das Wörterbuch E-VALBU umfasst neben einer Beschreibung der spezifischen Umgebung der verbalen Valenzträger auch Angaben zu deren semantischen Beschreibungen. Es richtet sich vor allem an Dozenten und Lehrbuchautoren im Bereich Deutsch als Fremdsprache. Deshalb orientiert sich die Verbauswahl, die vollständig aus VALBU übernommen wurde, an der Wortschatzliste des „Zertifikats Deutsch“ (ZD) des Goethe-Instituts. Im Laufe der Zeit wurde die Verbauswahl zwar um einige Verben aus dem Gebiet ,allgemein wissenschaftliches Vokabular“ ergänzt; im Vergleich zu den Wörterbüchern DUDEN und DWDS sind in E-VALBU bislang jedoch deutlich weniger Verben erfasst.

Die Lesartenunterscheidung erfolgt nach Auskunft der Autoren vor allem nach „semantischen Kriterien“. Entsprechend spielt auch hier die Nennung von Paraphrasen bzw. Synonymen eine entscheidende Rolle. Darüber hinaus werden - stärker als in anderen Wörterbüchern - auch Unterschiede in den Valenzbeziehungen $^{93}$ der Verwendungsweisen berücksichtigt. Unterschiede im Satzbauplan sowie bei der syntaktischen und semantischen Bestimmung von Komplementen werden u. U. als Indiz für kleinere bzw. größere Bedeutungsunterschiede der Verben gewertet, vgl. (88):

annehmen

i.S.v. $\quad$ jemand nimmt etwas, das angeboten wird, in Anspruch

Satzbauplan $\mathrm{K}_{\mathrm{SUB}}, \mathrm{K}_{\mathrm{AKK}}$ (s. E-VALBU_annehmen; 30.05. 2018) (4 $^{94}$

Die Anordnung der einzelnen Verwendungsweisen in E-VALBU entspricht noch der in VALBU. Zurzeit wird jedoch die Reihenfolge der Subartikel überarbeitet. Hierbei soll die Bedeutungsnähe der einzelnen Verwendungsweisen stärker berücksichtigt werden. Nach welchen Kriterien diese ermittelt wird, wird allerdings bisher nicht expliziert.

93 Der E-VALBU zugrundeliegende Valenzbegriff wird in Zifonun et al. (1997) vertreten.

$94<$ E-VALBU_annehmen; 30.05.2018> steht für die Angabe <E-VALBU 2018_annehmen, bereitgestellt am 30.05.2018 (URL: http://hypermedia.ids-mannheim.de/evalbu/index.html)>. Wird im weiteren Verlauf auf Wörterbucheinträge von E-VALBU verwiesen, wird ausschließlich die Kurzform (E-VALBU_[Verb; Abrufdatum]) verwendet. 


\subsection{Alternative Verfahren zur Ermittlung von Verwendungsweisen}

Um die unterschiedlichen Lesarten eines Ausdrucks zu erfassen, reichen die Angaben in den (o.g.) verschiedenen Wörterbüchern häufig nicht aus. Es zeigt sich, dass diese sowohl, was die Anzahl der voneinander abgegrenzten Bedeutungen als auch was deren Zusammenfassung zu einzelnen Unterlesarten angeht, zum Teil stark voneinander abweichen. Andererseits sind die vorgenommenen Bedeutungsdifferenzierungen nicht immer intuitiv nachvollziehbar. DUDEN und DWDS nehmen z. B. für das Verb akzeptieren je nur eine Lesart an, vgl. (86) und (87). In E-VALBU ist das Verb akzeptieren zwar nicht erfasst. In elexiko ${ }^{95}$, einem Online-Wörterbuch der deutschen Gegenwartssprache des Instituts für deutsche Sprache Mannheim, ist es aber analog zu den Darstellungen im DUDEN und DWDS ebenfalls mit nur einer Lesart enthalten, vgl. (89):

akzeptieren

i.S.v. jemand erklärt sich mit einem bestimmten Sachverhalt, einer Handlung oder einer Person(engruppe) einverstanden und erkennt diese( $n$ ) an (elexico_akzeptieren; 31.05.2018) ${ }^{96}$

Im Unterschied zu den genannten Wörterbüchern wird in dieser Arbeit Winkler (2009) folgend die Annahme vertreten, dass akzeptieren (mindestens) über die folgenden zwei Lesarten verfügt: akzeptieren $_{1}$ in der Bedeutung von jemand stimmt etwas zu und akzeptieren ${ }_{2}$ im Sinne von jemand nimmt etwas hin. Wie die Darstellungen in Kapitel 3.2 gezeigt haben, manifestieren sich die Bedeutungsunterschiede der beiden Lesarten in den je spezifischen semantischen und syntaktischen Valenzrahmen sowie unterschiedlichen Weglassungsbedingungen (vgl. (81) und (82)).

95 elexiko ist „ein Online-Informationssystem („Wörterbuch“) zur deutschen Gegenwartssprache, das den Wortschatz der deutschen Sprache anhand von aktuellen Sprachdaten (bis ins Jahr 2013) in sog. Modulen dokumentiert, erklärt und wissenschaftlich kommentiert. Der Schwerpunkt von elexiko liegt im Modul Lexikon zum öffentlichen Sprachgebrauch auf der ausführlichen Beschreibung von Bedeutung und Verwendung der (hochfrequenten) Stichwörter.“ (elexiko 2018_Start). Das Projekt wurde 2017 beendet.

96 <elexiko_akzeptieren; 31.05.2018> entspricht der Kurzform von <elexiko 2018_akzeptieren, bereitgestellt am 31.05.2018; URL: http://www.owid.de/artikel/114988>. Im Folgenden wird nur noch die Kurzform (elexiko_[Verb; Abrufdatum]) verwendet, wenn auf elexiko-Wörterbuchartikel verwiesen wird. 
Wenn also die Angaben in den Wörterbüchern nicht immer ausreichen, um die Lesarten eines Verbs hinreichend und nachvollziehbar $\mathrm{zu}$ bestimmen, wie lassen sich dann die Verwendungsweisen eines sprachlichen Ausdrucks mit Hilfe möglichst objektivierbarer Kriterien ermitteln?

Zunächst können die unterschiedlichen Lesarten eines sprachlichen Ausdrucks „normalsprachlich disambiguiert/vereindeutigt werden dadurch, daß man für jede von ihnen nahezu äquivalente/gleichwertige und explizierte Paraphrasen/Umformulierungen nachweist“ (von Polenz 2008³ 58). Tatsächlich gehört die Angabe von semantischen Paraphrasen und Synonymen ${ }^{97} \mathrm{zu}$ den verbreitetesten Verfahren zur Unterscheidung von divergenten Verwendungsweisen sprachlicher Ausdrücke (vgl. Fritz 2005: 21f.). In Kapitel 4.2.1 wird daher die Paraphrasenmethode dargestellt und auf ihre Wirksamkeit hin überprüft. Es wird sich zeigen, dass sie nicht nur zur Darstellung der unterschiedlichen Lesarten nützlich ist (vgl. Kapitel 3.2), sondern auch zur Ermittlung der für eine Verblesart bedeutungskonstitutiven Leerstellen.

Die verbbegleitenden Konstituenten sind mit lesartenspezifischen Forderungen und Restriktionen verbunden, die Gegenstand grammatischer und semantischer Valenzforderungen sind (vgl. Engelberg 2010: 114). Es stellt sich aber die Frage, wie sich diese (lesarten-)spezifischen syntaktischen und semantischen „Verwendungsregeln“ ermitteln lassen. Hierzu bedarf es einer Möglichkeit, aus dem Sprachgebrauch heraus die Kategorien abzuleiten und zu spezifizieren, mit denen die spezifischen Valenzforderungen einer Verblesart beschrieben werden können (vgl. Perkuhn et al. 2015: 6). Es stehen unterschiedliche traditionelle Verfahren zur Verfügung, die im Folgenden kurz skizziert werden sollen. Im Einzelnen handelt es sich dabei im Zusammenhang mit kategorialen Valenzforderungen vor allem um die Untersuchung syntaktischer Eigenschaften (vgl. Kapitel 4.2.2). Mit Blick auf semantische Valenzforderungen kommen zusätzliche Verfahren wie die Kollokations- bzw. Kolligationsanalyse zur Ermittlung sortal spezifischer Forderungen und Restriktionen (vgl. Kapitel 4.2.3) sowie Verfahren zur Überprüfung rollenspezifischer Postulate (vgl. Kapitel 4.2.4) zum Einsatz ${ }^{98}$.

97 Auch fremdsprachliche Äquivalente sowie Antonyme werden z. T. zur Bedeutungsdifferenzierung herangezogen (vgl. Fritz 2005: 21f.). Sie spielen für die nachfolgenden Überlegungen jedoch keine Rolle und werden daher nicht weiter betrachtet.

98 Ein weiteres Verfahren zur Unterscheidung von Verwendungsweisen ist der sog. Kipp-Test. Er basiert auf der Annahme, dass viele Sätze unterschiedliche Verständnismöglichkeiten aufweisen, zwischen denen die Deutung „,sozusagen hin und her schwankt“ (Fritz 2005: 21). So kann der Satz Er muss wissen, dass das nicht stimmt entweder im Sinne von Es gibt starke Indizien dafür, dass er weiß, dass das nicht stimmt oder im Sinne von Es besteht für ihn die Notwendigkeit zu wissen, dass das nicht stimmt gedeutet werden. Neben solch eindeutigen Fällen wie der Unterscheidung zwischen epistemischen und nicht-epistemischen Verwendungsweisen von müssen, lassen sich 


\subsubsection{Semantische Paraphrasen und Synonyme}

Das verbreitetste Verfahren zur Unterscheidung von Verwendungsweisen sprachlicher Ausdrücke ist die Angabe semantischer Paraphrasen ${ }^{99}$ (vgl. Fritz 2005: 21). Unter Bedeutungsparaphrasen versteht man im Allgemeinen die Umschreibung der Bedeutung eines Wortes mit Hilfe anderer sprachlicher Ausdrücke (vgl. Schwarz/Chur 2004 $: 34$ ). Dabei kann die Paraphrase ,aus einem einzelnen Wort bestehen, aus einem zusammengesetzten Ausdruck oder gar einem ganzen Satz“ (Klein 2015: 71).

Die semantische Paraphrase wird vor allem als umfassende Bedeutungserläuterung einer Lesart oder deren Spezifizierung eingesetzt (vgl. Storjohann 2005). Daher ist es nur konsequent, dass - ob im gedruckten Wörterbuch oder

jedoch auch zahlreiche Fälle erkennen, bei denen die Bedeutung nicht so offensichtlich kippt. Deshalb wird die Wirksamkeit des Kipp-Tests zur Bedeutungsdifferenzierung häufig angezweifelt. Darüber hinaus setzt eine Anwendung des Kipp-Tests m. E. die „Kenntnis der Art der sprachlichen Handlung, die mit der Verwendung der entsprechenden Äußerungsform gemacht werden kann, die Kenntnis des thematischen Zusammenhangs, das gemeinsame Wissen und das Wissen über bestimmte Gegenstände, Vorgänge, Handlungen usw.“ (Bons 2009: 32) voraus. Damit müssen für eine (erfolgreiche) Anwendung des Tests die möglichen Lesarten eines zu untersuchenden sprachlichen Ausdrucks bereits bekannt sein. Der Kipp-Test kann somit zwar zur Bestätigung bekannter, nicht jedoch zur Ermittlung neuer Verwendungsweisen herangezogen werden.

Ein weiterer Grund, die Nutzbarkeit dieses Kipp-Tests für die vorliegende Arbeit in Frage zu stellen, besteht darin, dass in Fällen, ,in denen wir die Vermutung mehrerer Verwendungsweisen auf unterschiedliche Kollokationen des betreffenden Ausdrucks stützen [...] der Kipp-Test prinzipiell nicht anwendbar“ ist (Fritz 2005: 21), vgl. Er spielt Tennis vs. Er spielt Wallenstein; ein Umstand, der vor allem für die hier zu untersuchenden Verben problematisch ist, da ihre Lesarten mit spezifischen semantischen und syntaktischen Forderungen an ihre Komplemente verbunden sind.

In der vorliegenden Arbeit ebenso unberücksichtigt bleibt der sog. Koordinations- oder Zeugma-Test. Hierbei wird zur Überprüfung, ob etwa spielen in den Sätzen Er spielt Tennis und Er spielt Wallenstein in unterschiedlichen Verwendungsweisen vorkommt, eine Koordination beider Objektargumente (hier: Tennis und Wallenstein) vorgenommen. Klingt diese Verknüpfung inkongruent, liegt also ein Zeugma vor, d. h. es handelt sich um zwei unterschiedliche spielen-Varianten; andernfalls geht es um das gleiche spielen. Tatsächlich klingt die Verknüpfung Er spielt morgens Tennis und abends Wallenstein seltsam, weshalb wir hier von zwei verschiedenen spielen-Lesarten der Sport-spielen- und der Theater-spielen-Lesart - ausgehen. Allerdings ist auch bei diesem Test eine eindeutige Trennschärfe nicht immer gegeben. In Fällen wie Ich liebe meinen Beruf und die Patienten herrscht häufig Uneinigkeit darüber, ob ein zeugmatischer Effekt vorliegt - wir also von zwei unterschiedlichen Verwendungsweisen ausgehen - oder nicht.

99 In der Lexikographie tauchen neben dem Begriff der Paraphrase auch andere Bezeichnungen, etwa Definition, Bedeutungsangabe, -erklärung, -beschreibung, -umschreibung, -paraphrase, -periphrase, -explikation, -kommentar oder lexikalische Paraphrase auf (vgl. Storjohann 2005: 183). 
im digitalen lexikalischen System - die Wortbedeutung im Kern durch Paraphrasen dargestellt wird (vgl. Klein 2015: 76). Auch im allgemeinen Sprachgebrauch hat die Bedeutungsparaphrase großen Wert, „da sie für den Spracherwerb, die sprachliche Kommunikation in der Schule und im beruflichen und alltäglichen Leben (Explikation, Auflösung von Uneindeutigkeiten usw.) unumgänglich ist“ (Lewandowski $1990^{5} \mathrm{~b}$ : 777).

In der vorliegenden Arbeit werden semantische Paraphrasen ebenfalls vor allem dazu eingesetzt, die unterschiedlichen Verwendungsweisen eines Verbs, die mit Hilfe anderer Verfahren ermittelt wurden, zu benennen und zu spezifizieren. Um „ein schnelles Erfassen der vorliegenden Einzelbedeutung [...] zu ermöglichen“ (Storjohann 2005: 199), werden - wo möglich und nötig - zusätzlich Synonyme angegeben. Als Synonyme werden hier verbale Ausdrücke verstanden, durch die die $\mathrm{zu}$ analysierenden Verbvarianten in jedem Kontext ausgetauscht werden können, ohne dass sich die Satzbedeutung dadurch wesentlich verändert (vgl. Schumacher et al. 2004: 58.).

Neben dem Einsatz als umfassende Bedeutungsspezifikation einer Lesart werden semantische Paraphrasen hier auch dazu genutzt, die unterschiedlichen Verwendungsweisen eines sprachlichen Ausdrucks zunächst einmal zu ermitteln. Semantische Paraphrasen in Wörterbüchern dienen vor allem dazu, so viele Informationen zu liefern, wie nötig sind,

um das (eventuell unbekannte) Lexem zu verstehen, um semantische Assoziationen hervorzurufen und um es Benutzer(inne)n zu ermöglichen, das Stichwort in lexikalisch-semantische Strukturen einordnen und v. a. um es angemessen gebrauchen zu können.

(Storjohann 2005: 183)

Entsprechend geht es bei Bedeutungsumschreibungen nach Harras (1991) eben nicht nur darum, die Bedeutung des betreffenden Wortes anzugeben. Wittgenstein geht davon aus, dass die Bedeutung eines Wortes sein Gebrauch in der Sprache ist (vgl. Wittgenstein 2001: § 43); deshalb müssen auch „,seine Beziehungen $\mathrm{zu}$ anderen Wörtern beschrieben werden, und zwar nur solche, die das zu beschreibende Wort in der vorliegenden Bedeutung aufweist“" (Storjohann 2005: 184). So stecken ,gerade in seiner kontextuellen Verwendung viele semantische Aussagen über ein Lexem“ (ebd. 186).

Folgt man einem solchen handlungsbezogenen Ansatz der Bedeutungsumschreibung, handelt es sich bei semantischen Paraphrasen nicht nur um reine Bedeutungserläuterungen bzw. Spezifizierungen einer Lesart, sondern auch um „Regelformulierungen (semantisch-pragmatischer oder syntaktischer Art) für den Gebrauch eines Lexems“ (ebd. 185). In diesem Sinne lassen Bedeutungsparaphrasen neben dem semantischen Inhalt im engeren Sinne auch die spezifischen bedeutungskonstitutiven Stellen der jeweiligen Verblesart erkennbar werden. 
Verdeutlichen wir uns diese Überlegungen an einem konkreten Beispiel: In einem Satz wie Peter öffnet eine Konservendose kann das zugrundeliegende Verb öffnen in der hier vorliegenden Lesart wie folgt paraphrasiert werden:

(90) öffnen

i.S.v. jemand bewirkt, dass etwas zugänglich ist; aufmachen

Eine solche Umschreibung lässt im Sinne der zuvor formulierten Bedingungen zweierlei Rückschlüsse zu: Zum einen geben sowohl die Bedeutungsparaphrase als auch das zusätzlich angegebene Synonym Auskunft über den „semantischen Inhalt“ der verwendeten öffnen-Lesart - das Verb öffnen bedeutet hier, dass etwas aufgemacht, d. h. zugänglich gemacht wird, damit es benutzt werden kann.

Darüber hinaus zeigen die in der Paraphrase enthaltenen Verwendungsangaben, in welchem Kontext das Verb in der dargestellten Lesart gebraucht werden kann. Gemeint sind sowohl syntagmatische als auch paradigmatische Beziehungsgeflechte, in denen „die Funktion des Stichwortes in einer Phrase oder in einem Satz deutlich“ wird (Storjohann 2005: 184). Bezeichnen wir z. B. einen Sachverhalt mit öffnen, nehmen wir damit (in der hier dargestellten Lesart) automatisch zwei Leerstellen an: Eine für die Bezeichnung des Partizipanten, der die Handlung des Öffnens durchführt, sowie eine weitere für den vom öffnen-Vorgang betroffenen Partizipanten (vgl. Primus 2015: 79):

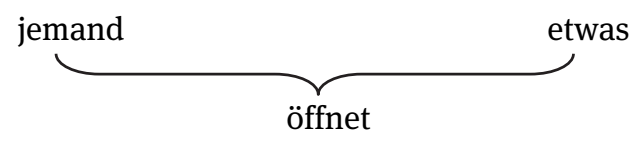

Beide vom „Verb eröffneten Stellen sind bedeutungskonstitutiv für das betreffende Verblexem“ (ebd.). Somit kann die oben genannte Bedeutungsumschreibung unter Berücksichtigung der genannten Verwendungsangaben insofern präzisiert werden, als öffnen in der hier dargestellten Lesart bedeutet, dass durch einen die Handlung des Öffnens durchführenden Partizipanten ein von der Handlung des Öffnens betroffener Partizipant aufgemacht, d.h. zugänglich gemacht wird, damit er benutzt werden kann. In diesem Sinne muss man sich auf die spezifischen von der Verblesart eröffneten Stellen beziehen, will man die besondere Verwendungsweise einer Verblesart verstehen und beschreiben.

Um möglichst alle Verwendungsweisen der darzustellenden Lesart zu erfassen, werden semantische Paraphrasen bewusst generalisierend abstrakt formuliert ${ }^{100}$,

100 In der Literatur wird darauf hingewiesen, dass in einsprachigen Wörterbüchern verschiedene, traditionell verankerte Erläuterungstypen zu finden sind, wobei viele „dieser Erläuterungs- 
wobei i.d.R. das besonders deutlich beschrieben wird, was Nutzer typischerweise mit der Lesart assoziieren (vgl. Storjohann 2005). Da die Bedeutungsparaphrase in der vorliegenden Arbeit neben der reinen Bedeutungsdarstellung auch der Ermittlung spezifischer Verwendungsweisen dient, wird auf die Nennung spezieller Einzelreferenzen i.d.R. bewusst verzichtet. Typische syntaktische und semantische Merkmale der verbbegleitenden Konstituenten können deshalb häufig erst in einem nachgelagerten Analyseschritt ermittelt werden.

Darüber hinaus wird versucht, „die Beschreibungssprache der Standardsprache zu entnehmen, damit die Bedeutungsbeschreibung nicht selbst wieder einer Erklärung bedarf“ (Schumacher et al. 2004: 58).

Zwar gelingt es mit Hilfe der Paraphrasenmethode in vielen Fällen relativ problemlos, die verschiedenen Verwendungsweisen eines sprachlichen Ausdrucks zu beschreiben. Tatsächlich reicht sie jedoch nicht aus, „um die semantischen Eigenschaften eines Wortes wirklich zu erfassen“ (Klein 2015: 73). Gängiger lexikographischer Praxis folgend werden in dieser Arbeit zusätzlich zur semantischen Paraphrase konkrete Beispiele für die Verwendungsweisen des jeweiligen sprachlichen Ausdrucks angegeben (vgl. ebd. 74). Durch die Beispiele soll „zugleich illustriert und bewiesen [werden, TvdB], wie das betreffende Wort in unterschiedlichen Zusammenhängen gebraucht wurde oder wird“ (ebd.). Sie dienen also dazu, die Bedeutung und Verwendung eines Lexems zu illustrieren und die in der Paraphrase enthaltenen Angaben zu plausibilisieren (vgl. Storjohann 2005: 195).

Außerdem werden die semantischen Paraphrasen - wie oben bereits angemerkt - für weitere Überlegungen und Analysen genutzt. Um die Bedeutung des Verbs bzw. der spezifischen Verblesart zu verstehen und zu beschreiben, können wir uns z. B. auf die vom Verb ausgewiesenen bedeutungskonstitutiven Stellen und ihre semantischen Rollen beziehen (vgl. Primus 2015: 79). Verben drücken „Beziehungen zwischen Personen und Sachen aus. Dabei sind diese Beziehungen von der jeweiligen Verbbedeutung semantisch festgelegt, so dass der Erwerb eines Verbs

typen [...] nicht starr voneinander abgegrenzt werden [können, TvdB] und [...] deshalb auch in Kombination miteinander“ (Storjohann 2005: 191) auftreten können.

Für die vorliegende Arbeit wird eine sehr allgemeine Formulierung für die Bedeutungsparaphrasen gewählt; anders als in Wörterbüchern werden sie vor allem dazu verwendet, die möglichen Lesarten eines Verbs zu ermitteln. Entsprechend sollen mit Hilfe der Bedeutungsparaphrasen zwar die für eine Verblesart bedeutungskonstitutiven Stellen erkennbar werden. Wie diese Stellen jedoch syntaktisch und semantisch zu besetzen sind, zeigen erst daran anschließende Untersuchungen. Die hier verwendete Paraphrase lässt sich daher nur schwerlich einem der zu findenden Erläuterungstypen (einsprachiger) Wörterbücher zuordnen, weshalb auf eine Darstellung und Erläuterung der einzelnen Typen an dieser Stelle verzichtet wird. Der interessierte Leser sei jedoch auf die Darstellungen und Überlegungen von Weber (1996) verwiesen. 
mit dem Erwerb seiner semantischen Rollen einhergeht. “(ebd. 80) In diesem Sinne bedeutet z. B. das Verb essen in einem Satz wie Peter isst einen Hamburger, dass eine belebte Person essbare Nahrungsmittel zu sich nimmt (vgl. u. a. DUDEN 20124). Wie wichtig semantische Rollen auch für metasprachliche, d.h. sprachreflexive Aufgaben sind und wie Verbbedeutungen mit ihrer Hilfe möglichst genau beschrieben werden können, wird in Kapitel 4.2.4 näher erläutert.

Ebenfalls von Bedeutung sind die mit den einzelnen Stellen verbundenen sortalen Forderungen und Restriktionen. Eine Möglichkeit, die sortalen Forderungen einer Verblesart zu ermitteln, ist die Kollokationsanalyse. Lesarten werden „oft dadurch angesetzt, dass ein Wort eine bestimmte Kollokation eingeht“ (Model 2010: 88). Welchen Nutzen dieses Verfahren auch für die vorliegende Arbeit hat, wird in Kapitel 4.2.3 dargestellt.

Neben Rollenforderungen und sortalen Forderungen und Restriktionen bestimmen die bedeutungskonstitutiven Stellen auch „die Zahl und Art der Satzglieder“ (Primus 2015: 79), weshalb in Kapitel 4.2.2 auf die Bedeutung syntaktischer Eigenschaften bei der Lesartenbestimmung eingegangen wird.

Trotz des großen Nutzens der Paraphrasenmethode darf nicht übersehen werden, dass sie mit einem nicht unerheblichen methodischen und erkenntnistheoretischen Problem verbunden ist: „Wir paraphrasieren das, was wir eigentlich erklären wollen, wieder mit etwas, was erklärungsbedürftig ist“ (Schwarz/Chur 2004: 34f.). Die zur Bedeutungserklärung herangezogenen Synonyme und Paraphrasenausdrücke sind i.d.R. selber polysem. Entsprechend müsste man, wenn man z. B. essen mit etwas zu sich nehmen paraphrasieren möchte, eigentlich auch angeben, wie man etwas zu sich nehmen verstanden wissen möchten - z. B. als Nahrung aufnehmen usw. (vgl. Bons 2009). Darüber hinaus weist Geeraerts (1993) auf ein weiteres Problem hin, indem er warnt, „that the polysemy or indeterminacy of a word may hide in the polysemy or indeterminacy of the words used to define it“ (Ravin/Leacock 2000: 4). Mit der gewählten Paraphrase wird immer ein bestimmter Verwendungsaspekt hervorgehoben, der - würde man eine andere Paraphrase wählen - möglicherweise verborgen bliebe. Fritz (1995) verdeutlicht dieses Problem anhand der Beispielsätze Dieses Messer ist scharf, Sein Verstand ist scharf, Dieser Pfeffer ist scharf und Diese Brille ist scharf:

Wählen wir zur Paraphrase dieser Sätze die Ausdrücke geschliffenes Messer, klarer Verstand, starker Pfeffer, leistungsstarke Brille, dann erscheint uns die Verwandtschaft vielleicht weniger eng, als wenn wir wählen gutes Messer, guter Verstand, guter Pfeffer, gute Brille.

(ebd. 81) 
Ähnliche Schwierigkeiten zeigen sich auch im Zusammenhang mit den hier zu untersuchenden Verben: Wählt man etwa für die Sätze Peter unterschrieb den Vertrag und Peter unterschrieb die Autogrammkarte die allgemeine Paraphrase seinen Namen unter etwas setzen, werden mögliche Bedeutungsunterschiede des Verbs unterschreiben in den hier vorliegenden Sätzen verdeckt. Denn Peter unterschrieb den Vertrag ist zu verstehen im Sinne von den Inhalt eines Schriftstücks bestätigen, wohingegen Peter unterschrieb die Autogrammkarte im Sinne von etwas signieren $\mathrm{zu}$ interpretieren ist.

Diese Überlegungen zeigen, dass es durchaus sinnvoll ist, „sich der Bedeutung von Äußerungen durch Umschreibungen zu nähern, Paraphrasen zu bilden“ (Hoffmann 2012: 16), um die von einer Verblesart eröffneten, bedeutungskonstitutiven Stellen zu ermitteln. Allerdings müssen sich daran weitergehende Analysen anschließen, um die spezifischen syntaktischen und semantischen Verwendungsbedingungen zu ermitteln.

\subsubsection{Untersuchung syntaktischer Eigenschaften}

Ausgehend von der Bedeutungsparaphrase ist die Untersuchung syntaktischer Eigenschaften der jeweiligen Verblesart eine erste Möglichkeit für weitere Analysen. So lassen die bedeutungskonstitutiven Stellen u. a. auch Rückschlüsse auf „die Zahl und Art der Satzglieder“ (Primus 2015: 79) zu.

Die Paraphrase in (92) zeigt, dass das Verb ziehen zwei bedeutungskonstitutive Stellen eröffnet: eine Stelle für die Bezeichnung des Partizipanten, der sich allmählich irgendwohin bewegt, sowie eine zweite Stelle für die Bezeichnung der direktiven Ortsangabe. Beide Stellen werden in der syntaktischen Valenz angegeben:

(92) ziehen $_{1}$

i.S.v. etwas gelangt, sich allmählich fortbewegend, irgendwohin (vgl. E-VALBU_ziehen_28.05.2018)

Bsp.: Die Wolken ziehen Richtung Westen. (TvdB)

SynVal: /nom /Ort (direktiv)

Es ist nun bekannt, dass die Argumentstrukturmuster vieler Verben variieren können und dass Änderungen in der Syntax häufig mit semantischen Differenzen einhergehen (vgl. Willems/Coene 2003: 38). So ergibt sich eine von (92) abwei- 
chende Verwendungsweise des Verbs ziehen allein aufgrund unterschiedlicher syntaktischer Merkmalsforderungen ${ }^{101}$, vgl. (93):

(93) ziehen $_{2}$

i.S.v. jemand/etwas bewegt jemanden/etwas unter Anwendung von Kraft hinter sich her (vgl. E-VALBU_ziehen; 28.05.2018)

Bsp.: $\quad$ Der Esel zieht den Wagen. (TvdB)

SynVal: /nom /akk

Wie lassen sich die unterschiedlichen Argumentstrukturmuster eines Verbs ermitteln? Eine Möglichkeit hierzu bieten die sog. Kookkurrenz- und Kolligationsanalysen, bei denen das Kontextverhalten von sprachlichen Ausdrücken untersucht wird. Während als Kookkurrenz das reine „gemeinsame Vorkommen zweier Wörter in einem gemeinsamen Kontext betrachtet“ (Lemnitzer/Zinsmeister 20153: 179) wird, werden als Kolligationen „Paare sprachlicher Einheiten bezeichnet, deren Zusammenhang durch die Beziehung ihrer syntaktischen Kategorien und der Beziehungen zwischen diesen Kategorien weiter qualifiziert ist“ (ebd. 32). Ausführliche Darstellungen hierzu finden sich in Kapitel 4.2.3.

Vor allem der Kolligationsanalyse kommt bei der hier durchzuführenden Ermittlung der unterschiedlichen Argumentstrukturmuster eines Verbs eine zentrale Bedeutung zu. Bei der Feststellung möglicher Satzstrukturmuster geht es nicht nur um die reine Erfassung, Auszählung und häufigkeitsbasierte Ordnung von Wortpaaren, sondern vielmehr auch um die Herausarbeitung der jeweiligen grammatischen Beziehungen zwischen den Elementen (vgl. ebd.).

Zur Abfrage entsprechender Kolligationsbeziehungen eignet sich vor allem das DWDS-Wortprofil der berlin-brandenburgischen Akademie der Wissenschaften ${ }^{102}$. Hierbei handelt es sich um „eine auf syntaktischen Analysen und statisti-

101 Selbstverständlich unterscheiden sich die beiden Verwendungsweisen $z_{i e h e n}$ und ziehen $_{2}$ auch hinsichtlich ihrer semantischen Forderungen. Da es hier jedoch nur um die Unterschiede auf syntaktischer Ebene geht, werden die semantischen Forderungen an dieser Stelle nicht dargestellt.

$102 \mathrm{Da}$, wo die Ergebnisse lückenhaft oder widersprüchlich erscheinen, wird ergänzend eine Kookkurrenzanalyse in COSMAS-II durchgeführt. Die so ermittelten Analyseergebnisse können entweder in Form einer reinen Auflistung oder in Form syntagmatischer Muster dargestellt werden, so dass auch hier die unterschiedlichen Satzstrukturmuster, in denen ein Verb auftritt, ermittelt werden können (vgl. die Darstellungen in Kapitel 4.2.3). 
schen Auswertungen basierende automatische Kollokationsextraktion“ (Geyken 2011a: 9), die

einen kompakten Überblick über statistisch signifikante und damit typische Wortverbindungen (liefert, TvdB). Beispiele hierfür sind Attribut-Nomen-Verbindungen wie schöne Bescherung oder Verb-Objekt-Verbindungen wie Flasche entkorken.

(DWDS; cf. Wörterbuch)

Es ist damit ein System, das automatisch die relevanten syntaktischen Beziehungen eines Verbs und damit seine unterschiedlichen Argumentstrukturmuster anzeigt $^{103}$. Die Ergebnisse werden zum einen in Form einer Schlagwolke geliefert; zum anderen ist auch die Darstellung in der klassischen Tabellenform möglich, vgl. exemplarisch den Auszug aus dem Wortprofil zu ziehen ${ }^{104}$ in Abb. 3:

\begin{tabular}{lrr} 
Uberblick & logDice Ii & Freq. 1 I \\
\hline 1. zurückziehen & 9.8 & 41118 \\
\hline 2. anziehen & 9.7 & 37365 \\
\hline 3. Konsequenz & 8.8 & 19787 \\
\hline 4. einziehen & 8.8 & 18734 \\
\hline 5. vorziehen & 8.6 & 16392 \\
\hline 6. abziehen & 8.2 & 12313
\end{tabular}

\begin{tabular}{lrr}
\hline hat Akk./Dativ-Objekt & logDice 1 i & Freq. 1 i \\
\hline 1. Konsequenz & 11.4 & 16637 \\
\hline 2. Bllanz & 10.6 & 9054 \\
\hline 3. Schluß & 10.3 & 7676 \\
\hline 4. Lehre & 9.3 & 3714 \\
\hline 5. Notbremse & 9.0 & 2862 \\
\hline 6. Schlußstrich & 9.0 & 2744
\end{tabular}

\begin{tabular}{|lrr|}
\hline hat Präpositionalgruppe & logDice Ii & Freq. I? \\
\hline 1. in Betracht & 9.2 & 5638 \\
\hline 2. in Mitleidenschaft & 9.0 & 4580 \\
\hline 3. in Erwägung & 8.9 & 4201 \\
\hline 4. zur Rechenschaft & 8.8 & 4061 \\
\hline 5. aus Verkehr & 8.8 & 4130 \\
\hline 6. in Zweifel & 8.8 & 4012 \\
\hline
\end{tabular}

\begin{tabular}{|c|c|c|}
\hline hat Subjekt & logDice l'i & Freq. 14 \\
\hline 1. Familie & 7.6 & 1548 \\
\hline 2. Konsequenz & 7.5 & 1072 \\
\hline 3. Bilanz & 7.4 & 990 \\
\hline 4. Demonstrant & 6.9 & 762 \\
\hline 5. er & 6.8 & 27753 \\
\hline 6. sie & 6.7 & 22828 \\
\hline
\end{tabular}

Abb. 3: Wortprofil zu ziehen, Screen-Ausschnitt (DWDS_WP_ziehen; 28.05.2018).

Ein solch klares 1:1-Verhältnis zwischen Syntax und Semantik, wie die Beispiele in (92) und (93) suggerieren, ist jedoch nicht immer gegeben. Vielmehr können -

103 Auch in Sketch Engine ist eine auf syntaktischen Beziehungen basierende Kookkurrenzanalyse implementiert (vgl. URL: https://www.sketchengine.eu/; Abfrage 02.10.2019). Das Verfahren kommt in der vorliegenden Arbeit jedoch nicht zur Anwendung.

104 Daten, die über das DWDS-Wortprofil ermittelt werden, sollen zitiert werden als <DWDSWortprofil für „ziehen“, erstellt durch das Digitale Wörterbuch der deutschen Sprache, <https:// $w w w . d w d s . d e / w p / z i e h e n>$, abgerufen am 28.05.2018>. Der Übersichtlichkeit halber werden Belege dieser Quelle im weiteren Verlauf mit folgendem Kurztitel zitiert: DWDS_WP_[Verb; Abrufdatum]. 
wie im Falle von spielen in (94) und (95) - einem Satzmuster auch unterschiedliche Lesarten entsprechen:

(94) spielen $_{1}$

i.S.v. jemand musiziert auf etwas (vgl. E-VALBU_spielen; 29.05.2018)

Bsp.: $\quad$ Heiner spielt Gitarre. (TvdB)

SynVal: /nom /akk

(95) spielen $_{2}$

i.S.v. jmd. stellt etw. dar (vgl. E-VALBU_spielen; 29.05.2018)

Bsp.: $\quad$ Heiner spielt den Hamlet. (TvdB)

SynVal: /nom /akk

Andererseits können bei gleicher Lesart auch unterschiedliche Satzmuster vorliegen, wie die Beispiele in (96) und (97) zeigen $^{105}$ :

(96) suchen $_{1}$

i.S.v. jemand/etwas sieht irgendwo nach etwas/etwas Verlorenem, Verstecktem o. ̈. nach (vgl. E-VALBU_suchen; 29.05. 2018)

Bsp.: $\quad$ Paula sucht ihren Schlüssel. (TvdB)

SynVal: /nom /akk

(97) suchen $_{1}$

i.S.v. jemand/etwas sieht irgendwo nach etwas/etwas Verlorenem, Verstecktem o. A. nach (vgl. E-VALBU_suchen; 29.05. 2018)

Bsp.: $\quad$ Paula sucht nach ihrem Schlüssel. (TvdB)

SynVal: /nom /Prp ${ }_{\text {nach }}$

Sätze wie diese machen deutlich, dass eine Analyse syntaktischer Eigenschaften nicht immer ausreicht, um die möglichen Verwendungsweisen eines Verbs zweifelsfrei und allumfänglich zu ermitteln. Es müssen also weitere Kriterien herangezogen werden, um bei entsprechenden Verben die möglicherweise vorhandene Mehrdeutigkeit aufzulösen.

Eine Möglichkeit bietet die Kollokationsanalyse (vgl. Fritz 2005: 23), mit deren Hilfe „sich das typische lexikalische Kontextverhalten eines Wortes aufdecken“ (Perkuhn et al. 2015: 6) lässt.

105 Es gibt auch Annahmen, wonach jegliche syntaktische Veränderung automatisch auch zu einem mehr oder weniger großen Lesartenunterschied führt. 


\subsubsection{Kollokations- und Kolligationsanalyse}

Die Kollokationsmethode basiert auf der Beobachtung, dass sich Wörter beim Erzeugen und Verstehen von Sätzen nicht nur selber einbringen, sondern auch Einfluss auf das Verstehen und die Auswahl der Wörter in ihrer Umgebung haben (vgl. Perkuhn et al. 2012: 110). Tatsächlich zeichnen sich die unterschiedlichen Verblesarten durch je spezifische sortale Forderungen und Restriktionen für ihre Partizipanten aus, wie die Überlegungen zum Verb akzeptieren in Kapitel 3.2 zeigen konnten. Vor diesem Hintergrund können die Nuancen des Gebrauchs einer Verblesart durch Sammlung und Ordnung typischer Kollokationspartner ermittelt werden. Es überrascht deshalb nicht, dass Lesarten oft dadurch angenommen werden, dass ein Wort eine bestimmte Kollokation mit einem anderen Wort eingeht (vgl. Model 2010: 88).

Wurden Wortverbindungen lange Zeit vor allem im Bereich der Phraseologie untersucht, sind sie seit einiger Zeit auch in den Fokus anderer Forschungsrichtungen der Linguistik - etwa der Korpuslinguistik oder der Kollokationsforschung - gerückt. „Aber es gibt nicht die Richtung oder die Schule oder gar die Wortverbindungstheorie.“ (Steyer 2004: 89) Vielmehr zeichnet sich insbesondere die Kollokationsforschung durch eine sehr große Heterogenität aus, die in der Vergangenheit zu zahlreichen Missverständnissen geführt hat.

Ganz allgemein bezeichnet der Begriff der Kollokation ${ }^{106}$ „das sprachliche Phänomen, dass manche Wörter bevorzugt mit bestimmten anderen vorkommen“ (Steinbügl 2005: 3). Mit Blick auf die Forschungsliteratur zeigt sich jedoch, dass der Begriff keineswegs klar und eindeutig verwendet wird. Vielmehr lassen sich unterschiedliche Auffassungen ${ }^{107}$ erkennen, die im Extremfall Kollokationen entweder rein empirisch-quantitativ - d. h. statistisch geprägt (vgl. z. B. Sinclair et al. 2004) - oder rein qualitativ - d. h. z. B. bedeutungsorientiert (vgl. z. B. Hausmann 1984) - betrachten ${ }^{108}$.

106 Zur Entstehung und Entwicklung des Kollokationsbegriffs s. u. a. Cedillo (2004), Steinbügl (2005) und Steyer (2008).

107 Eine weitere, hier nicht beachtete Kollokationsauffassung wird von Halliday/Hasan (1976) vertreten. In ihrem textlinguistisch geprägten Ansatz gehen sie davon aus, dass Kollokationen sich dadurch auszeichnen, ,dass sie einen wesentlichen Beitrag zur Kohäsion leisten. Mit Kohäsion ist dabei die sprachliche Verknüpfung eines Textes gemeint, die beispielsweise auch dadurch entsteht, dass einzelne Textelemente wiederholt oder durch Pro-Formen erneut aufgegriffen werden. Eine Kollokation liegt dann vor, wenn bestimmte Wörter aufgrund eines äußeren Sachzusammenhangs gemeinsam vorkommen.“ (Steinbügl 2005: 15)

108 Während qualitativ ausgerichtete Kollokationsauffassungen nur die typischen, usuellen, konventionellen und rekurrenten Wortverbindungen als Kollokationen betrachten, erfassen empirisch-quantitativ ausgerichtete Ansätze auch die normalen syntaktischen und semanti- 
Die Differenzen zwischen den einzelnen Kollokationsdefinitionen führen häufig zu Missdeutungen. Um diese zu vermeiden, wird im Rahmen dieser Arbeit Hausmanns Forderung nach einer terminologischen Präzisierung (vgl. Hausmann 2004: 320f.) nachgekommen und im Folgenden zwischen Kollokationen (im engeren Sinne) und Kookkurrenzen unterschieden. Denn der Begriff der Kookkurrenz hat gute Chancen, „sich als ein weites Konzept für das statistisch signifikante Kovorkommen sprachlicher Einheiten im deutschsprachigen Raum durchzusetzen“ (Steyer 2008: 188). In diesem Sinne bezeichnen wir

- als Kookkurrenz „das gemeinsame Vorkommen zweier oder mehrerer Wörter in einem Kontext von fest definierter Größe“ (Kunze/Lemnitzer 2007: 319). Hierbei sollte das „gemeinsame Vorkommen [...] höher sein, als bei einer Zufallsverteilung aller Wörter erwartbar wäre“ (ebd.). Eine Kookkurrenz ist also ein berechnetes Phänomen. (vgl. Steyer 2008: 188)

- als Kollokationen (i.e.S.) linguistisch interpretierte Kookkurrenzen. Das heißt, dass Kollokationen neben den o.g. Kriterien eine innere Struktur in Form eines (semantisch) hierarchischen Determinationsverhältnisses zwischen Kollokationsbasis und Kollokator aufweisen ${ }^{109}$ (vgl. Lemnitzer/Zinsmeister 2015²: 179). Kollokationen sind somit als interpretierte Teilmenge des berechneten Kookkurrenzpotenzials zu verstehen. (vgl. Steyer 2004: 99)

Nach diesen Definitionen ist das Beispiel in (98) aufgrund des häufigen Kovorkommens als Kookkurrenz aufzufassen. Im Gegensatz dazu handelt es sich bei dem Beispiel in (99) um eine Kollokation, da hier ein semantisches Abhängigkeitsverhältnis zwischen der Kollokationsbasis Haare und dem Kollokator graue besteht:

(98) besteht aus (TvdB)

(99) graue Haare (ebf.)

Neben Kookkurrenzen und Kollokationen werden häufig auch sog. Kolligationen unterschieden. Im Unterschied zu Kollokationen, „where specific lexical but

schen Verträglichkeiten als Kollokationen, und zwar auch dann, wenn sie nicht typisch oder usuell sind (vgl. Cedillo 2004: 31, FN 20).

109 Darüber hinaus wird häufig darauf hingewiesen, dass „die Glieder einer Kollokation in einer syntaktischen Beziehung zueinander stehen, z. B. als Köpfe einer Verbalphrase und einer gleich- oder untergeordneten Nominalphrase, oder als Kopf einer Nominalphrase und Kopf einer untergeordneten Adjektivphrase“ (Lemnitzer/Zinsmeister 2015: 179).

Zur begrifflichen Präzisierung wird auf diesen Aspekt an dieser Stelle verzichtet. Stattdessen werden Kombinationen aus lexikalischen Ebenen und syntaktischen Ebenen als Kolligation bezeichnet (vgl. ebd. 32). 
not syntactic affinities for co-occurrence are relevant, colligation refers to both lexical and syntactic cohesion that exists between items in a syntagmatic string“ (Storjohann 2010: 1). Entsprechend bezeichnen

- Kolligationen „Paare sprachlicher Einheiten [...], deren Zusammenhang durch die Beziehung ihrer syntaktischen Kategorien und der Beziehung zwischen diesen Kategorien weiter qualifiziert ist“. (Lemnitzer/Zinsmeister 2015³ 32)

Unter Kolligationen werden also auch präferierte Umgebungen eines Wortes verstanden; allerdings nicht bezogen auf das lexikalische Material, sondern auf Wortstellung und grammatische Kategorien:

„Ein Wort bevorzugt oder meidet eine bestimmte Position innerhalb eines Syntagmas, es bevorzugt oder meidet Wörter in der Umgebung bestimmter grammatischer Kategorien und auch für ein Syntagma als Ganzes wird eine grammatische Funktion präferiert oder abgelehnt.“

(Perkuhn et al. 2012: 113)

In diesem Sinne handelt es sich bei dem Beispiel in (100) um eine Kolligation, da zwischen den beiden Elementen eine grammatische Beziehung - hier Prädikat und Objekt - vorliegt ${ }^{110}$ :

(100) Apfel essen (ebf.)

Für die nachfolgenden Überlegungen spielen vor allem das Kookkurrenz- sowie das Kolligationskonzept eine wichtige Rolle. Es gilt, für eine fundierte Lesartenunterscheidung die Kategorien zu ermitteln und zu kategorisieren, mit denen die Valenzleerstellen eines Verbs bevorzugt gefüllt werden.

Hierzu werden im Sinne einer Kookkurrenzanalyse zunächst alle sprachlichen Ausdrücke, die bevorzugt mit der zu untersuchenden Verblesart in einem Satz auftreten, ermittelt. Daran anschließend werden im Sinne einer Kolligationsanalyse die lexikalischen Einheiten herausgefiltert, die die von den jeweiligen Verblesarten ausgewiesenen Valenzleerstellen besetzen (können).

Um Kookkurrenzen zu ermitteln, stehen unterschiedliche Abfragesysteme zur Verfügung: Etwa das Wortschatzportal der Universität Leipzig, die in COSMAS-II implementierte Kookkurrenzanalyse des Instituts für deutsche Sprache Mannheim sowie das DWDS-Wortprofil der berlin-brandenburgischen Akademie der Wissen-

110 Die Beispiele in (98) und (99) können auf Grundlage dieser Definition ebenfalls als Kolligationen analysiert werden: in (98) wird eine Präposition von einem Verb regiert, in (99) liegt die attributive Erweiterung einer minimalen Nominalphrase vor. 
schaften. Allen Abfragesystemen ${ }^{111}$ gemeinsam ist, dass sie die einzelnen Stichwörter auf der Basis umfangreicher Korpora untersuchen und linguistische Details korpusgestützt ermitteln. Damit soll sichergestellt werden, dass die lexikografischen Angaben nicht allein auf der Basis von Introspektion einzelner Lexikografen gewonnen werden, sondern dass Wörter im Kontext, d.h. Sprache im Gebrauch, analysiert wird (vgl. Storjohann 2015: 106). Je nach Ausrichtung und Arbeitsgrundlage der einzelnen Abfragesysteme ergeben sich jedoch auch Unterschiede, die einen mehr oder weniger großen Einfluss auf die hier angestrebte Lesartenunterscheidung sowie die anschließend durchzuführende Analyse semantischer Konsequenzen bei Nicht-Realisierung einer Argumentstelle haben. Vor allem die Möglichkeit zur Kolligationsanalyse ist nicht immer gegeben. Deshalb sollen die drei genannten Systeme zur Kookkurrenzbestimmung im Folgenden anhand des Beispiels akzeptieren kurz vorgestellt und hinsichtlich ihrer Nutzbarkeit für diese Arbeit bewertet werden:

\subsubsection{Wortschatzportal}

Das Projekt Deutscher Wortschatz wird seit 1998 an der Universität Leipzig entwickelt. Es baut zu einzelnen Sprachen ${ }^{112}$ umfangreiche Textkorpora auf, wertet diese statistisch aus und stellt die Ergebnisse auf der Web-Seite http://wortschatz. uni-leipzig.de/de (Stand: 30.05.2018) zur Verfügung. Das primäre Ziel besteht darin, Verfahren zur Verarbeitung von Text zu entwickeln, die unabhängig von der zu untersuchenden Sprache funktionieren (vgl. Quasthoff/Richter 2005: 33).

Für das Deutsche können mittlerweile über neun Millionen verschiedene Wörter und Wortgruppen nachgeschlagen werden. Datengrundlage bilden vor

111 Ein weiteres Verfahren ist die Kookkurrenzdatenbank CCDB von Cyril Belica. Sie wurde 2001 am Institut für deutsche Sprache Mannheim entwickelt als eine „korpuslinguistische Denk- und Experimentierplattform, ein transparentes Labor zur Erforschung, Entwicklung und Evaluierung einer Methodik zur datengeleiteten Exploration und Modellierung des Sprachgebrauchs“ (CCDB 2012). Ziel der Forscher ist es, Einsichten in die Strukturen, Gesetzmäßigkeiten, Eigenschaften und Funktionen von Sprache zu erlangen, wobei ein derzeitiger Forschungsschwerpunkt auf der Analyse wechselseitiger Beziehungen zwischen Kookkurrenzprofilen liegt. Über die WebSeite http://corpora.ids-mannheim.de/ccdb/ (Stand: 31.05.2018) sind große Teile der CCDBDatenbank als eine Art <gläsernes Labor $>$ öffentlich zugänglich.

Im Kern enthält die CCDB große Mengen sekundärer empirischer Daten, d.h. Daten, „die durch mehrfache, unterschiedlich parametrisierte Kookkurrenzanalysen des gesamten Grundvokabulars eines großen Korpus errechnet“ (Belica 2011: 162) wurden. Die Kookkurrenzanalyse erfolgt nach dem in COSMAS-II implementierten Analyseverfahren, weshalb theoretisch alle CCDB-Kookkurrenzprofile über das COSMAS-II-System rekonstruiert und nachvollzogen werden können. Die Methode findet daher hier keine Anwendung.

112 Den Nutzern des Wortschatzportals stehen Korpora für über 250 Sprachen zur Verfügung (vgl. Wortschatzportal; cf: Korporaportal). 
allem Sätze aus der aktuellen überregionalen Tagespresse mit besonderem Schwerpunkt ab ca. 1995 (vgl. ebd.: 34). Für jeden Eintrag stellt das Wortschatzportal Häufigkeitsangaben und Beispielsätze zur Verfügung. Darüber hinaus werden bei den Ergebnissen neben grammatischen Informationen (Wortart, Geschlecht und Flexion) und Informationen zu Sachgebieten und Synonymen auch Kookkurrenzpartner, also statistisch signifikant vorkommende Partnerwörter, angegeben. Dies geschieht einerseits auf der Basis ganzer Sätze (Satzkookkurrenzen), andererseits für typische unmittelbare linke und rechte Nachbarn des Ausgangswortes (Nachbarschaftskookkurrenzen) (vgl. ebd.), vgl. exemplarisch die Übersicht in Abb. 4 für das Verb akzeptieren ${ }^{113}$ :

- Kookkurrenzen:

nicht $(4,369)$, zu $(3,269)$, . $(3,257)$, dass $(2,289)$, muss $(1,548)$, bereit $(1,426)$, müssen $(1,291)$, wir $(1,250)$, sie $(872)$, man $(751)$, werc (597), wollen (564), die (560), Entscheid (531), diese (522), dies (517), nicht bereit (512), Wenn (472), würden (452), aber (442), sag (349), schwer (319), Urteil (317), keine (288), andere (280), wollte (279), es (257), sollte (256), müssten (252), verstehen (250), als (:

- Signifikante linke Nachbarn:

zu (14,786), nicht (7,638). Wir (527), einfach (287), nicht mehr (230), man (221), niemals (193), wir (191), nicht länger (172), bereit zt (128), Urteil (123), Entscheid (103), länger (103), müssen (102), will nicht (99), Niederlage (94), ohne weiteres (89), endlich (89), dies kann nicht (69), nie (68), Schlichterspruch (68), muss (65), keinesfalls (65), Visa-Partner (64), nicht einfach (63), das (62), Regeln (6z Gewinnspiels (57), auch (57), Gesetze (56), Meinungen (55), Auflagen (55), Mehrheitsentscheidung (55), Opel-Eigner (54), Grünen (

- Signifikante rechte Nachbarn:

(7,920), , (4,604), und (727), können (549), ? (392), kann (388), würde (381), müssen (363), würden (332), wollen (317), w (228), wc wollten (69), könne (68), " (48), bereit (45), wolle (38), könnten (37), konnte (37), - (36), könnte (30), Sie (28), keine (27), müsse (26), (15), willst (15), ; (13), möchte (13), mussten (12), gelernt (11), mögen (9.7), sie (9.5), - (9.5), wir auch (8.9), gelte (8.8), dürfen (7.3)

Abb. 4: Kookkurrenzen und signifikante Nachbarn zu akzeptieren, Screen-Ausschnitt (WoSchaPo_akzeptieren; 31.05.2018).

Für jedes Ausgangswort werden maximal 50 Kookkurrenzpartner ermittelt, wobei Funktionswörter, zu denen auch Satzzeichen zählen, ebenfalls in die Analyse miteinbezogen werden. Die Kookkurrenzdaten werden automatisch $\mathrm{zu}$ einer bestimmten Wortform ermittelt. Eine Lemmatisierung, d. h. die Zusammenfassung aller Wortformen, die auf dasselbe Lemma zurückgeführt werden können, ist dagegen ausgeschlossen. Deshalb müssen mehrere Suchanfragen durchgeführt werden, will man die unterschiedlichen Gebrauchsvarianten eines Verbs ermitteln. Gesucht werden im Rahmen dieser Arbeit alle indikativischen Flexionsformen des jeweiligen Suchwortes - für akzeptieren im Einzelnen akzeptiere,

113 Daten aus dem Wortschatzportal werden im Folgenden zitiert als WoSchaPo_[Verb; Abrufdatum]. 
akzeptierst, akzeptiert, akzeptieren, akzeptierte, akzeptiertest, akzeptierten und akzeptiertet ${ }^{114}$.

Die so ermittelten signifikanten Kookkurrenzpartner, bei denen die Stärke ihrer Zusammengehörigkeit in Klammern angegeben wird, sind das Ergebnis eines reinen Rechercheprozesses, weshalb zur Überprüfung möglicher Kolligationen eine nachgelagerte Interpretation der Daten erforderlich ist. Hierbei ist zu prüfen, welche der Kookkurrenzpartner welche Valenzleerstellen des Suchwortes bevorzugt besetzen. Das Wortschatzportal stellt Verwendungsbeispiele des Verbs zur Verfügung, die zu einer entsprechenden Analyse genutzt werden können, vgl. Abb. 5:

- Beispiele:

- Beim Elterngeld musste Schröder Abstriche akzeptieren. (www.bkz-online.de, gecrawlt am 23.12.2010)

- Er sei bereit, Zardari als Präsidentschaftskandidaten zu akzeptieren. (wnw.news.ch, gecrawit am 16.01.2011)

- Selbstironie ist herrlich, wenn alle sie akzeptieren. (umw.pnp.de, gecrawit am 19.01.2011)

- Den Platz auf der Tribüne, so lässt er durchblicken, würde er ohne Murren akzeptieren. (www.bernerzeilung.ch, gecrawit am 22.12.2010)

- Das Land hatte sich dagegen gewehrt, eine steigende Durchschnittstemperatur zu akzeptieren. (www.20min.ch, gecrawit am 18.12.2010)

- Ein Abstimmungsverhalten in einer geheimen Wahl muss man akzeptieren. (wuw.ff-online.de, gecrawit am 31.12.2010)

- Sie akzeptieren die Niederlage nicht und fordern die Neuauszăhlung aller Stimmen. (www.net-news-global.de, gecrawit am 13.01.2011)

Abb. 5: Verwendungsbeispiele zu akzeptieren, Screen-Ausschnitt (WoSchaPo_akzeptieren; 31.05.2018).

Bei den Verwendungsbeispielen handelt es sich um automatisch erhobene Sätze, die nicht mit den ermittelten Kookkurrenzpartnern verknüpft sind. Aus diesem Grund ist oft nicht eindeutig erkennbar, ob, und wenn ja, welche Valenzstelle des Verbs das jeweilige Partnerwort besetzt. Vielmehr lassen sich die syntagmatischen Bezüge häufig nur erraten (vgl. Geyken 2011b: 117). Wegen dieser unsicheren Aussagekraft kommt dieses Verfahren im Rahmen dieser Arbeit nicht zur Anwendung.

\subsubsection{Kookkurrenzanalyse in COSMAS-II}

Das seit 1964 aufgebaute Deutsche Referenzkorpus ${ }^{115}$ (DeReKo) des Instituts für deutsche Sprache Mannheim (IDS) ist mit mehr als 42 Mrd. Wörtern (Stand: April 2018) „die weltweit größte linguistisch motivierte Sammlung elektroni-

114 Für die Formen akzeptiertest und akzeptiertet sind im Wortschatzportal keine Daten vorhanden.

115 Auf das DeReKo wird häufig auch mit anderen Bezeichnungen - etwa Mannheimer Korpora, IDS-Korpora, COSMAS-Korpora, Archiv der Korpora geschriebener Gegenwartssprache - verwiesen. Die Bezeichnung DeReKo wurde ursprünglich nur für einen Teil des heutigen Gesamtarchivs verwendet; seit 2004 ist sie die offizielle Bezeichnung für das gesamte Korpusarchiv. 
scher Korpora mit geschriebenen deutschsprachigen Texten aus der Gegenwart und der neueren Vergangenheit“ (IDS_Korpuslinguistik 2018). Es umfasst neben Zeitungstexten auch belletristische, wissenschaftliche und populärwissenschaftliche Texte sowie eine breite Palette weiterer Textsorten aus Deutschland, Österreich, Luxemburg und der Schweiz. Das Korpus wird kontinuierlich weiterentwickelt; es trägt damit „dem Auftrag des IDS Rechnung, den Gebrauch der deutschen Gegenwartssprache fortlaufend $\mathrm{zu}$ dokumentieren“ (Lüngen 2012: 26).

Das Corpus Search, Management and Analysis System II (COSMAS-II ${ }^{116}$ ) erlaubt, das DeReKo nach linguistischen Kriterien zu durchsuchen. COSMAS-II liegt in zwei Varianten vor: eine reine Internet-Anwendung mit einer zeilenorientierten Anfragesprache und eine auf Microsoft Windows laufende Anwendung, die zusätzlich über eine graphische Anfragesprache verfügt. Für die vorliegende Arbeit wird die webbasierte Version verwendet. Diese Volltextdatenbank steht der Öffentlichkeit über die Web-Seite http://www.ids-mannheim. de/cosmas2/ (Stand: 30.05.2018) nach einer kostenlosen Registrierung zur Verfügung ${ }^{117}$.

Ausgangspunkt einer jeden Analyse ist die Erfassung des Vorkommens eines Suchwortes im Korpus (vgl. Steyer 2003: 218). Daher ist zunächst eine auf das jeweilige Forschungsinteresse abgestimmte Korpus- und Archivauswahl ${ }^{118} \mathrm{zu}$ treffen. Eine COSMAS-II-Recherche wird immer

in einem sogenannten aktiven Korpus durchgeführt. Das aktive Korpus wird dadurch bestimmt, dass man noch vor der Suche einen Eintrag aus der Liste der vordefinierten virtuellen Korpora wählt oder ein benutzerdefiniertes virtuelles Korpus lädt. (COSMAS-II; cf. COSMAS-II Projekt Textorganisation> Virtuelle Korpora)

Für diese Arbeit wird i.d.R. auf das vordefinierte Archiv W-Archiv der geschriebenen Sprache zurückgegriffen. Als Korpus wird $W$ - öffentlich verwendet; es

$116<$ COSMAS I/II (Corpus Search, Management and Analysis System), http://www.ids-mannheim.de/cosmas2/, (c) 1991-2016 Institut für Deutsche Sprache, Mannheim> wird im Folgenden zitiert als <COSMAS-II $>$.

117 Aus urheber- und lizenzrechtlichen Gründen sind Teile des DeReKo-Materials nur IDSintern zugänglich. Der überwiegende Teil (ca. 95\%) ist jedoch zu wissenschaftlichen Zwecken über die o. g. Webadresse recherchierbar (vgl. IDS 2018b).

118 Archive stellen eine Art Sammelstelle für verschiedene Korpora dar, wobei die Aufteilung der „Korpora in verschiedene Archive [...] die Unterschiede in der Zusammensetzung, im Bearbeitungszustand, im Format und in anderen grundlegenden Merkmalen der Korpora“ (COSMAS-II; cf. COSMAS-II $\mathrm{I}_{\text {Projekt }}>$ Textorganisation $>$ Archive) widerspiegelt. 
umfasst alle öffentlichen Korpora des Archivs W inklusive aller Neuakquisitionen. Die Archiv- und Korpusauswahl ist dadurch begründet, dass, um alle Lesarten eines Verbs ausmachen zu können, möglichst viele Verwendungsweisen der zu untersuchenden Verben in unterschiedlichen Verwendungskontexten ermittelt werden müssen ${ }^{119}$. Daher wird nur in Ausnahmefällen eine Eingrenzung der zu erhebenden Daten z. B. über die Textsorte vorgenommen. Entsprechende Angaben hierzu finden sich bei den einzelverbbezogenen Untersuchungen in Kapitel 5.

In einem zweiten Schritt sind für das jeweilige Forschungsvorhaben passende Suchanfragen $\mathrm{zu}$ formulieren. Die Rechercheergebnisse können dann nach unterschiedlichen Kriterien - etwa Entstehungszeit, Erscheinungsort oder Thematik - sortiert werden. Darüber hinaus können die Suchergebnisse auch mittels einer Kookkurrenzanalyse gesichtet, sortiert und tabellarisch dargestellt werden. Bei der Formulierung einer Suchanfrage ist darauf $\mathrm{zu}$ achten, dass die Wortformen, die Gegenstand einer anschließenden Kookkurrenzanalyse sein sollen,

den eindeutigen Kern des Trefferobjektes ausmachen. Dies gilt trivialerweise für die Suche nach einer bestimmten Wortform oder nach den Wortformen, denen dieselbe Grundform (oder derselbe reguläre Ausdruck) zugrundeliegt. Bei komplexeren Suchanfragen [...] ist dies nicht garantiert.

(Perkuhn/Belica 2004)

Ziel der hier angestrebten Kookkurrenzanalyse ist, Mehrdeutigkeiten von Verben aufzulösen. Kookkurrenzen liefern häufig wichtige Hinweise darauf, wie die (semantischen) Valenzleerstellen einer Verblesart bevorzugt gefüllt werden. Deshalb werden zunächst alle Flexionsformen eines Suchwortes im Korpus ermittelt. COSMAS-II stellt hierfür den sog. Grundformoperator ,\&‘ zur Verfügung, der es ermöglicht, Flexionsformen ihren Grundformen zuzuordnen. Die mit Hilfe des Lemmatisierungsverfahrens ermittelten Wortformen werden in einer temporären Wortformenliste zusammengefasst, vgl. Abb. 6 .

119 Die so erhobenen Daten enthalten Belege deutschsprachiger Texte aus Deutschland, Österreich, Luxemburg und der Schweiz. Eine Einschränkung der Belege auf deutsche Quellen wurde vor dem Hintergrund, dass bereits die Belege aus Deutschland alle (Dialekt-)Regionen abdecken, nicht vorgenommen. Zudem ergaben verbspezifische Ergebnispräsentationen sortiert nach Ländern (D/CH/A/L), dass Belege aus Deutschland zahlenmäßig mit Abstand am stärksten vertreten sind - vgl. z. B. bei 10.000 Zufallstreffern für das Verb akzeptieren entfallen 63 auf Luxemburg, 1.334 auf Österreich, 1.631 auf die Schweiz und 6.972 auf Deutschland. 


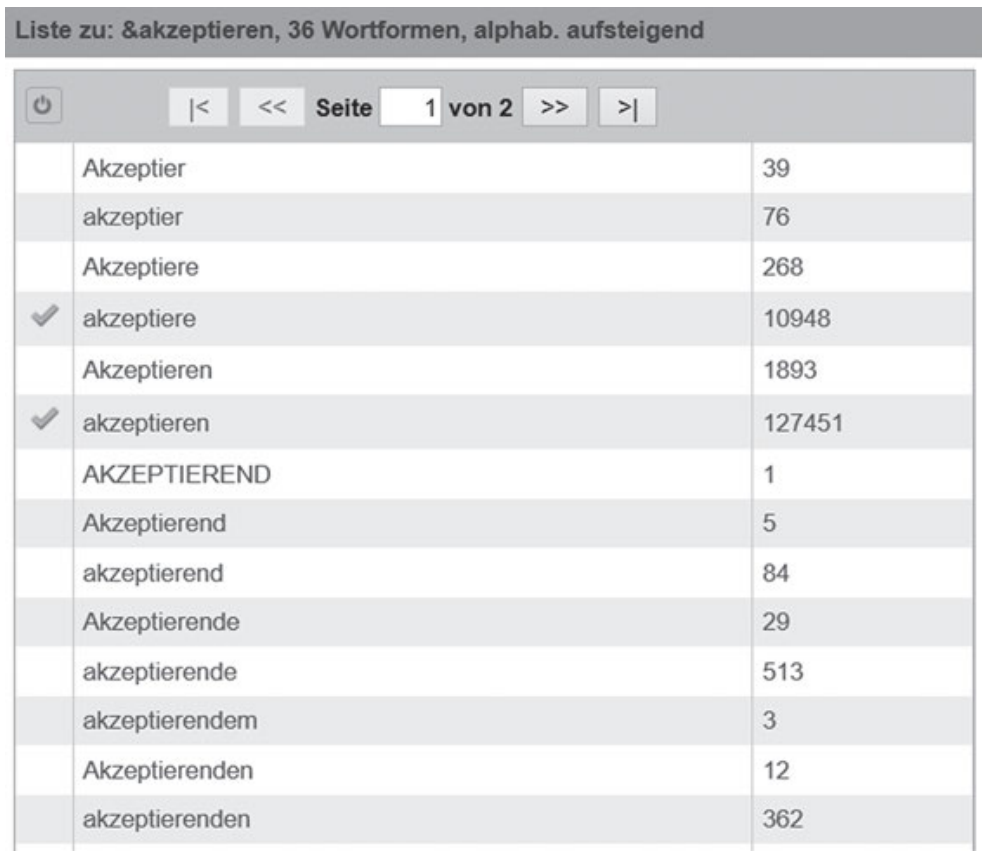

Abb. 6: Wortformenliste zu \&akzeptieren, Screen-Ausschnitt (COSMAS-II).

Abbildung 6 zeigt einen Auszug aus der temporären Wortformenliste zu \&akzeptieren. Sie umfasst 36 Wortformen, wobei neben den hier $\mathrm{zu}$ untersuchenden verbalen Flexionsformen auch zahlreiche „Fehlbelege“ - etwa adjektivische Formen wie akzeptierenden oder substantivische Verwendungen wie Akzeptieren - enthalten sind. Diese müssen zunächst deaktiviert werden. Übrig bleiben schließlich sieben verbale Wortformen - im Einzelnen akzeptiere, akzeptieren, akzeptierst, akzeptiert, akzeptierte, akzeptierten und akzeptiertest ${ }^{120}$.

Die Key-Word-in-Context-Belege (KWICs), d.h. die zeilenweise präsentierten Kontexte des Bezugswortes aller Trefferobjekte der Suchanfrage sind Ausgangspunkt für die anschließende Kookkurrenzanalyse (vgl. Perkuhn/Belica 2004). In Abb. 7 ist ein Auszug der 280.309 Belege für die Suchanfrage \&akzeptieren dargestellt.

120 Die Form akzeptiertet fehlt hierbei; sie ist - analog zum Wortschatzportal - mit keinem Beleg im Korpus enthalten. 


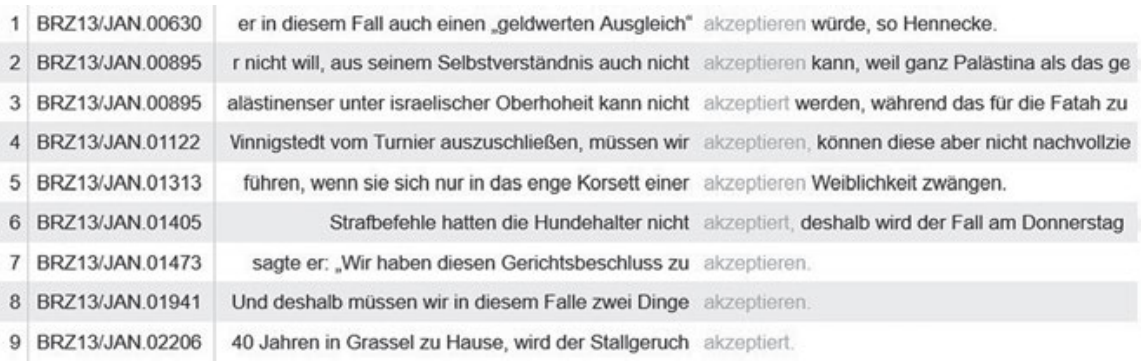

Abb. 7: KWIC zu \&akzeptieren, Screen-Ausschnitt (COSMAS-II).

Mit Hilfe des in COSMAS-II implementierten Analysemoduls ${ }^{121}$ werden anschließend automatisch die Wörter herausgefiltert, die einen statistisch auffälligen Kohäsionsgrad zum Suchwort haben. Dabei geht es nicht um Frequenz, d.h. um ein zahlreiches Vorkommen eines Wortes in der Nähe des Suchwortes, sondern um statistische Auffälligkeiten. Statistisch auffällig können danach auch Wörter sein, die im Korpus insgesamt eher selten vertreten sind, jedoch signifikant oft in der Nähe des Suchwortes auftauchen (vgl. Steyer 2004: 95f.).

Suchwort und Kookkurrenzpartner bilden das sog. Kookkurrenzcluster. Strukturen aus Suchwort und primärem Kookkurrenzpartner stellen die Basisrelationen dar. Da solche binären Strukturen häufig nicht ausreichen, um die wirkliche Bedeutung und den Gebrauch einer sprachlichen Einheit zu ermitteln, werden in einem nächsten Schritt weitere Kookkurrenzpartner in der Nähe dieser Cluster gesucht. Diese sukzessive Kontextspezifikation erfolgt so lange, bis keine weiteren (multiplen) Kookkurrenzpartner mehr gefunden werden. Die Gesamtheit aller Kookkurrenzcluster ergibt das Kookkurrenzpotenzial eines Wortes.

Die Kookkurrenzanalyse ist kein starres Verfahren. Vielmehr eröffnet es zahlreiche Möglichkeiten der Parametervariation, wodurch Nutzer - je nach Erkenntnisinteresse - unterschiedliche Analyseergebnisse erzeugen können (vgl. hierzu ausführlich Perkuhn/Belica 2004). So kann neben der Korpus- und Archivauswahl sowie der Suchanfrage auch das auszuwertende Kontextfenster an die jeweilige Forschungsfrage angepasst werden. Darüber hinaus lassen sich auch Parameter beeinflussen, die die Vorgehensweise bei der Analyse steuern - etwa die Lemmatisierung (bezogen auf die Wortformen im Kontext des Suchwortes) oder der Ausschluss von Funktionswörtern. Ebenfalls einstellbar ist der Grad der Kontextsensitivität der Analyse. Für alle Parameter werden von den Autoren Standardwerte vorgeschlagen, deren Beibehaltung sich für die vorliegende Arbeit

121 Das hierzu in COSMAS-II implementierte Analysemodul basiert auf der von Belica entwickelten Analysemethode Statistische Kollokationsanalyse und -clustering (Belica 1995). 
bewährt hat. Gesucht werden Kookkurrenzpartner, die im gleichen Satz maximal fünf Wörter links bzw. rechts vom jeweiligen Suchwort auftauchen. Die Wortformen im Kontext des Suchwortes werden hierbei einzeln ausgewertet, und nicht wie bei eingeschalteter Lemmatisierung - alle Wortformen gemeinsam, die auf dasselbe Lemma zurückgeführt werden können. Funktionswörter wie Artikelwörter, Pronomen, Präpositionen, Konjunktionen und Satzzeichen bleiben von der Analyse zunächst ${ }^{122}$ ausgeschlossen. So soll verhindert werden, dass die in der Sprache und somit auch in Korpora häufig vorkommenden Wörter andere und für diese Arbeit u. U. aussagekräftigere Kookkurrenzpartner verdrängen.

Die Analyseergebnisse enthalten neben Angaben zu Häufigkeiten und Stärke der Zusammengehörigkeit die ermittelten Kookkurrenzpartner in Form einer reinen Auflistung sowie in Form syntagmatischer Muster, vgl. Abb. 8. Die entsprechenden Belege sind als KWIC- oder Volltextanzeige hinterlegt:

\begin{tabular}{|c|c|c|c|c|c|c|}
\hline 田 & & & 100662 & 561 & Strafbefehl & $38 \%$ einen|den Strafbefehl [ ... ] akzeptiert \\
\hline 田 & 11 & 6377 & 111709 & 11047 & wird & $50 \%$ akzeptiert [...] wird \\
\hline 田 & 12 & 5783 & 113274 & 1565 & bereit & $80 \%$ bereit [ . zu] akzeptieren \\
\hline 田 & 13 & 5540 & 115456 & 2182 & würden & $35 \%$ akzeptieren [...] würden \\
\hline 田 & 14 & 5376 & 115467 & 11 & Entscheid gefallten & $\begin{array}{l}81 \% \text { den demokratisch gefallten Entscheid [zu] } \\
\text { akzeptieren und }\end{array}$ \\
\hline 田 & & & 116321 & 854 & Entscheid & $38 \%$ den Entscheid [ . . zu] akzeptieren \\
\hline 田 & 15 & 5287 & 118996 & 2675 & würde & $40 \%$ akzeptieren [. . . w würde \\
\hline 田 & 16 & 5266 & 119445 & 449 & zähneknirschend & $39 \%$ zähneknirschend [...] akzeptiert \\
\hline 田 & 17 & 4160 & 131724 & 12279 & auch & $26 \%$ auch [ ... zu] akzeptieren \\
\hline 田 & 18 & 3915 & 131728 & 4 & Zahlungsmittel Rubel & $50 \%$ Rubel ... als Zahlungsmittel [...] akzeptiert \\
\hline 田 & & & 132034 & 306 & Zahlungsmittel & $48 \%$ als Zahlungsmittel [ ... ] akzeptiert \\
\hline 田 & 19 & 3767 & 134370 & 2336 & kann & $49 \%$ kann [ich ...] akzeptieren \\
\hline 田 & 20 & 3681 & 134382 & 12 & Bedingungen gestellten & $50 \%$ gestellten [. . B Bedingungen [fur zu] akzeptieren \\
\hline 田 & & & 135191 & 809 & Bedingungen & $41 \%$ die Bedingungen [... zu] akzeptieren \\
\hline 田 & 21 & 3580 & 136342 & 1151 & müsse & $76 \%$ musse [man ... akzeptieren dass \\
\hline
\end{tabular}

Abb. 8: Kookkurrenzprofil zu \&akzeptieren, Screen-Ausschnitt (COSMAS-II).

Bei den Ergebnissen handelt es sich um Resultate interpretationsfreier Rechercheprozesse (vgl. Steyer 2004: 98). Um zu bestimmen, welche der 3.685 ermittel-

122 Bei einzelnen Verben wird eine zweite Suchanfrage, in der Funktionswörter im oben definierten Sinne mit ermittelt werden, durchgeführt, weil insbesondere präpositionale Anschlüsse häufig die Annahme weiterer Lesarten legitimieren:

(V) a. Peter glaubt Susi.

b. Peter glaubt an Susi.

Während (Va) i.S.v. davon überzeugt sein, dass jemand die Wahrheit sagt zu interpretieren ist, bedeutet (Vb) so viel wie davon überzeugt sein, dass jemand große Fähigkeiten besitzt. 
ten Kookkurrenzpartner für die hier angestrebte Lesartenunterscheidung relevant sind, bedarf es einer nachgelagerten Interpretation (vgl. Perkuhn et al. 2015: 6). So ist es erforderlich - will man die Mehrdeutigkeiten eines Verbs auflösen -, genau die Kategorien abzuleiten, mit denen die Valenzleerstellen einer bestimmten Verblesart bevorzugt gefüllt werden. Für eine Kolligationsanalyse stehen in COSMAS-II keine Analysewerkzeuge zur Verfügung. Einzig die Angaben zu syntagmatischen Mustern (s. o.) können hier als Anhaltspunkte dienen.

Um die für die weitere Analyse relevanten Partnerwörter zu ermitteln, müssen zunächst alle Kookkurrenzpartner, die keine valenzrelevante Stelle des Verbs besetzen können - etwa Angaben dazu, wie etwas akzeptiert wird: zähneknirschend, einstimmig, mehrheitlich, weitgehend usw. -, aus der Liste entfernt werden. Anschließend wird überprüft, inwieweit die restlichen Korpusbefunde „das abdecken, was man als Wissen über dieses Lexem bereits kodifiziert hat, und ob [...] Kontextualisierungen rekonstruierbar sind, die uns das Wörterbuch nicht anbietet“ (Steyer 2002: 223). Zum Vergleich seien die Lexikoneinträge der Wörterbücher DUDEN, DWDS und elexiko zu akzeptieren in (101) bis (103) dargestellt:

(101) DUDEN 2012 ${ }^{4}$ (Hervorhebungen durch Fettdruck ergänzt, TvdB) akzeptieren $\approx$ annehmen, hinnehmen, billigen; anerkennen; mit jemandem oder etwas einverstanden sein - eine Entschuldigung akzeptieren; der Vorschlag wurde von allen akzeptiert; sie wurde [als Chefin] von allen akzeptiert; er akzeptierte schließlich, dass er zurückstehen musste; er akzeptierte (nahm das Angebot an)

(102) DWDS_eWDG_akzeptieren; 30.05.2018 (Hervorhebungen id.) akzeptieren $\approx$ etw. annehmen - ein Angebot, einen Vorschlag, eine Bedingung akzeptieren

(103) elexiko_akzeptieren; 30.05.2018 (Hervorhebungen id.)

Mit akzeptieren bezeichnet man eine Handlung, bei der sich eine Person(engruppe) mit einem bestimmten Sachverhalt (z.B. einem Vorschlag), einer Handlung (z. B. einer Entscheidung) oder einer anderen Person(engruppe) einverstanden erklärt und diesen oder diese anerkennt.

Die Darstellungen in den drei Wörterbüchern zeigen, dass bei der Lesartenunterscheidung von akzeptieren vor allem die Objektvalenzstelle von besonderer Bedeutung ist. Während sich die Subjektvalenzstelle stets auf (im weitesten Sinne) Personen oder Institutionen bezieht, finden sich in den Bedeutungspa- 
raphrasen bzw. Beispielen der Wörterbücher für die Objektvalenzstelle Ausdrücke wie z. B. Vorschläge, Angebote, Entscheidungen, Bedingungen sowie Personen (gruppen). Bei genauerer Betrachtung der genannten Partnerwörter zeigt sich, dass die aufgeführten Objekttypen keineswegs einheitlich sind. Vielmehr zeichnen sie sich durch eine weitreichende Heterogenität aus, die sich mit Blick auf die mit COSMAS-II ermittelten signifikanten Kookkurrenzpartner noch zu verstärken scheint:

Bei der Interpretation der Analyseergebnisse sind viele Kookkurrenzpartner hervorgetreten, die im weitesten Sinne eine Art „Angebot“ beschreiben (z. B. Vorschläge, Pläne, Offerten und Entwürfe). Diesen Objekten ist gemeinsam, dass sie entscheidungsoffen sind; sie können willentlich angenommen oder abgelehnt werden. Im Vordergrund steht die bewusste Einflussnahme, Entscheidung oder Wahlmöglichkeit dessen, womit man sich einverstanden erklärt. Sie tragen daher das Merkmal [+ENTSCHEIDUNGSOFFEN].

Im Gegensatz dazu steht eine Gruppe von Partnerwörtern, die gerade nicht entscheidungsoffen sind. Sie beschreiben eher eine Art ,unabänderlichen Sachverhalt“ (z. B. Schicksale, Tatsachen, Entscheidungen und Urteile), weshalb bei ihnen ein bewusstes Zustimmen oder Ablehnen dessen, was akzeptiert wird, ausgeschlossen ist. In diesem Sinne tragen sie das Merkmal [-ENTSCHEIDUNGSOFFEN].

Neben diesen beiden Verwendungsweisen lassen sich weitere Befunde ausmachen, bei denen sich die Kookkurrenzpartner von akzeptieren auf eine Art „Zahlungsmittel“ beziehen (z. B. Bargeld, EC-Karten, Währungen und Tickets). Sie tragen das Merkmal [+FINANZ].

Wiederum andere Kookkurrenzpartner beziehen sich auf komplexe Konzepte, die gedanklich konzeptionelle Verschiedenartigkeiten beschreiben: Deren Abweichung - etwa in Bezug auf die eigene Meinung oder soziale Norm - kann erkannt und in Folge zugelassen/geduldet werden (z. B. Meinungen, Argumente, Kritik, Unterschiedlichkeit und Vielfalt). Sie tragen daher das Merkmal [+VERSCHIEDEN Z.B. IN BEZUG AUF DIE EIGENE MEINUNG, SOZIALE NORMEN].

Davon abzugrenzen sind Partnerwörter, die umfassende Konzepte beschreiben, die eine (institutionelle) Wirkungsmacht über das Subjekt entfalten (u.a. Regierungen, Gesetze, Werte und staatliche Souveränität). Im Unterschied zu den zuvor beschriebenen Kookkurrenzpartnern steht hier die Anerkennung von Höherrangigkeit dessen, was akzeptiert wird, im Vordergrund. Sie signalisieren eine Art „Unterwerfungsbereitschaft“ und tragen daher das Merkmal [+HÖHERRANGIG Z.B. IN BEZUG AUF DIE EIGENE PERSON].

Neben den genannten fünf Lesarten haben die hier durchgeführten Kookkurrenz- und Kolligationsanalysen zwei weitere Verwendungsweisen des Verbs akzeptieren erkennbar werden lassen: akzeptieren, dass und akzeptieren als. Sie 
ergeben sich bereits auf der syntaktischen Ebene, wie die unterschiedlichen syntaktischen Valenzen zeigen, vgl. (104) und (105):

(104) akzeptieren, dass

Bsp.: $\quad$ Paula akzeptiert, dass Moritz sie nicht nach Berlin begleiten will. (TvdB)

synVal: /nom /dass

\section{(105) akzeptieren als}

Bsp.: $\quad$ Moritz akzeptiert sie als Chefin. (ebf.)

synVal: /nom /akk/als

Da diese Verwendungsweisen des Verbs akzeptieren für die nachfolgenden Überlegungen keine Rolle spielen - die in der Literatur genannten sortalen Effekte können sich nicht auf diese spezifischen Nutzungen beziehen - werden sie in der nachfolgenden Systematisierung nicht erfasst; auch eine weitere Analyse, z. B. zu möglichen Kookkurrenzpartnern, wird nicht vorgenommen.

Die Übersicht auf S. 113 zeigt eine Systematisierung dieser Beobachtungen, vgl. Abb. 9.

Viele Kookkurrenzpartner lassen sich den oben beschriebenen Lesarten relativ einfach eindeutig zuordnen. Ein Angebot beschreibt - im Sinne von Lesart $_{1}$ - immer etwas Entscheidungsoffenes, eine Tatsache - im Sinne von Lesart $_{2}$ - einen gegebenen Umstand, Bargeld bezeichnet - im Sinne von Lesart ${ }_{3}-$ ein Zahlungsmittel, eine Meinung beschreibt - im Sinne von Lesart ${ }_{4}$ - eine persönliche Ansicht und ein Gesetz bezieht sich - im Sinne von Lesart ${ }_{5}$ - auf eine bindende Vorschrift.

Bei einigen Kookkurrenzpartnern ist die Zuordnung $\mathrm{zu}$ den beschriebenen Gruppen jedoch nicht immer offensichtlich. Das liegt darin begründet, dass die entsprechenden Ausdrücke selber polysem, und damit hinsichtlich ihrer Bedeutung stark kontextabhängig sind: So kann sich der Begriff Vertrag im Sinne von Lesart $_{1}$ auf einen Entwurf, vgl. (106a) oder im Sinne von Lesart ${ }_{5}$ auf eine Art Gesetz, vgl. (106b), beziehen:

(106) a. Daheim wurde der Vertrag zwischen Deutschland und Österreich attackiert, weil nationale Interessen zu wenig berücksichtigt wurden. In Brüssel jedoch haben sie ihn akzeptiert.

b. Der Versailler Vertrag entspricht nicht in allen Punkten der Ansicht der aktuellen Bundesregierung, trotzdem akzeptiert sie ihn. 


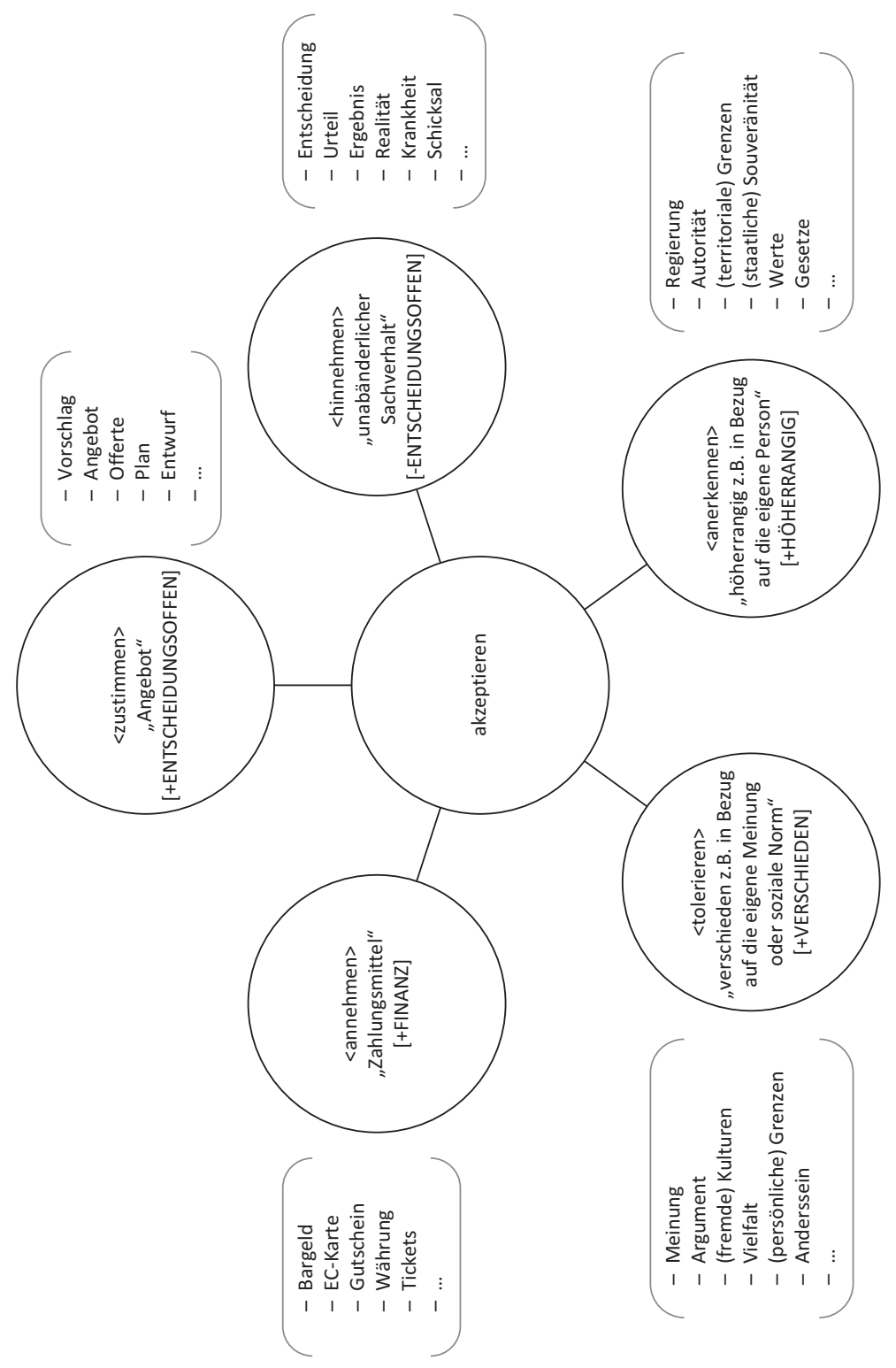

Abb. 9: Ergebnisübersicht der Kookkurrenzanalyse zu akzeptieren. 
Um dieser Beobachtung zu entsprechen, werden die jeweiligen Kookkurrenzpartner - gemäß ihrer jeweiligen kontextuellen Bedeutung - $u$. U. mehreren Lesarten zugeordnet.

Mit Hilfe dieses mehrschrittigen Analyseverfahrens des IDS lässt sich das Kontextverhalten der hier zu untersuchenden Verben gut ermitteln und darstellen. Über die Kookkurrenz- und Kolligationspartner können zudem Rückschlüsse auf die unterschiedlichen Verwendungsweisen der Verben gezogen werden. Das Verfahren wird daher im weiteren Verlauf falls notwendig eingesetzt.

\subsubsection{DWDS - Wortprofil}

DWDS - das digitale Wörterbuch der deutschen Sprache wurde an der berlinbrandenburgischen Akademie der Wissenschaften entwickelt und steht der Öffentlichkeit über die Web-Seite www.dwds.de (Stand: 04.05.2018) zur Verfügung. Es verfolgt das Ziel, umfassende Auskunft über den deutschen Wortschatz in Gegenwart und Vergangenheit zu geben. Hierzu stehen den Nutzern unterschiedliche Ressourcen - etwa die an der berlin-brandenburgischen Akademie der Wissenschaften erarbeiteten Wörterbücher und Korpora - zur Verfügung (s. Kapitel 3).

Eine Kernfunktion des DWDS ist das sog. Wortprofil, „eine auf syntaktischer Analyse und statistischer Auswertung basierende automatische Kollokationsextraktion“ (Geyken 2011a: 9). Zu diesem Zweck ermittelt das DWDS-Wortprofil statistisch saliente Kookkurenzen in einem lemmatisierten Korpus (vgl. Geyken et al. 2009: 143). Im Unterschied zu den zuvor beschriebenen Abfragesystemen werden diese Kookkurrenzen nach syntaktischen Relationen geordnet (vgl. ebd.). So liefert das Wortprofil einen kompakten Überblick darüber, mit welchen anderen Wörtern das Abfragewort häufig gemeinsam auftritt, und in welchen syntaktischen Relationen die Wörter dabei stehen (vgl. DWDS; cf. statistische Auswertungen). Es ermöglicht damit als einziges System ohne nachgelagerte Interpretation Aussagen sowohl zu Kookkurrenz- als auch zu Kolligationspartnern des jeweiligen Suchwortes.

Das Wortprofil basiert auf einer Mischung aus Referenz- und Zeitungskorpora ${ }^{123}$ und umfasst 5,5 Mrd. öffentlich recherchierbare laufende Textwörter (Stand 2018). Die Errechnung eines Profils erfolgt in drei Etappen: Zunächst sind die zu extrahierenden syntaktischen Relationen festzulegen, wobei sowohl phrasen-

123 Über DWDS stehen sog. Referenzkorpora zur Verfügung. Hierbei handelt es sich um zeitlich und nach Textsorten sorgfältig gewichtete Korpora, deren Textgrundlage literarische Werke, wissenschaftliche Texte, Gebrauchstexte und Zeitungstexte sind, die in einem ausgewogenen Verhältnis zueinander stehen (vgl. DWDS; cf. Textkorpora). Darüber hinaus sind aufgrund von Nutzungsvereinbarungen mit Verlagen unterschiedliche Zeitungskorpora - namentlich: Berliner Zeitung, Der Tagesspiegel, Die Zeit, Potsdamer Neueste Nachrichten, Welt, Bild sowie Süddeutsche Zeitung - verfügbar (vgl. ebd.). 
interne Relationen - etwa attributive Erweiterungen einer Nominalphrase (z. B. grüne Hose) - als auch phrasenübergreifende Relationen - etwa Prädikat/Objekt (z. B. den Apfel essen) - berücksichtigt werden (vgl. Geyken 2011b: 119). In einem nächsten Schritt werden die einzelnen Relationen mit Hilfe einer automatisch syntaktischen Analyse ${ }^{124}$ extrahiert und anschließend hinsichtlich ihrer statistischen Salienz bewertet. Da das Wortprofil Auskunft über die statistisch signifikanten syntagmatischen Beziehungen eines Wortes mit anderen Wörtern geben soll, bezieht die Salienz allerdings ,nicht die relativen Häufigkeiten über das gesamte Korpus mit ein, sondern schränkt diese auf den spezifischen syntaktischen Relationstyp ein, über den die Wortkombination in Verbindung steht“ (ebd. 122).

Abgefragt werden können über das Wortprofil nur einfache Wörter. Komplexe Suchanfragen - bezüglich Phrasen o. Ä. - sind hingegen ausgeschlossen. Gehört das Abfragewort mehr als einer Wortart an, muss festgelegt werden, nach welcher Wortart das Wortprofil suchen soll. Ausgewählt werden können Verben, Substantive, Adjektive, Adverbien und Personalpronomen. Als Abfragewort ist die jeweilige Grundform - bei Verben z. B. der Infinitiv - anzugeben. Aufgrund einer voreingestellten Lemmatisierung werden dann alle Wortformen, die auf dieselbe Grundform zurückgeführt werden können, abgebildet.

Das Wortprofil liefert zunächst einen Überblick über die Wörter mit der stärksten statistischen Assoziation; dieser umfasst alle syntaktischen Relationen (vgl. DWDS; cf. statistische Auswertungen). Die Analysen der einzelnen Relationstypen lassen sich dann über das Wortprofil-Fenster ansteuern, indem man die einzelnen Relationsfilter anklickt. Wählt man beispielsweise mit Blick auf akzeptieren den Relationstyp Akkusativ-/Dativobjekt, erhält man alle Partnerwörter, die die Objektvalenzstelle dieses Verbs besetzen können. Voraussetzung für die Berücksichtigung eines Partnerwortes im Wortprofil ist das Vorliegen von mindestens vier Belegen, um so zu verhindern, dass rein okkasionelle Verbindungen in das Wortprofil aufgenommen werden.

Die Ergebnisse werden zum einen in Form einer Schlagwortwolke geliefert, vgl. Abb. 10. Besonders signifikante Begriffe werden hierbei in einer größeren Schriftart präsentiert als weniger signifikante. Dabei basiert Signifikanz auf der oben erwähnten Salienz: „[...] je salienter eine syntaktische Relation, desto größer die Schriftart“ (ebd. 214). Die Partnerwörter werden demnach „über die reine statistische Signifikanz auch nach der syntaktischen Relevanz gewichtet“ (ebd.125). Abgebildet wird die im Korpus am häufigsten realisierte Flexionsform:

124 Das hierzu verwendete Analysewerkzeug ist SynCoP. Einen Überblick hierzu findet sich in Geyken (2011b). Eine vollständige technische Beschreibung des Verfahrens ist Didakowski (2008) zu entnehmen. 


\begin{tabular}{|c|c|c|c|c|c|c|c|}
\hline \multicolumn{6}{|c|}{ Lemma } & \multirow{2}{*}{\multicolumn{2}{|c|}{$\begin{array}{l}\text { optional: Wortve } \\
\text { Unterschied }\end{array}$}} \\
\hline \multicolumn{6}{|c|}{ akzeptieren } & & \\
\hline \multicolumn{2}{|c|}{ Wortart } & \multicolumn{2}{|c|}{$\min . \log$ Dice } & \multicolumn{2}{|c|}{$\min$. Frequenz } & \multicolumn{2}{|c|}{ Sortierung } \\
\hline Verb & $\checkmark$ & 0 & $\div$ & 5 & $\div$ & logDice & $\checkmark$ \\
\hline
\end{tabular}

Überblick
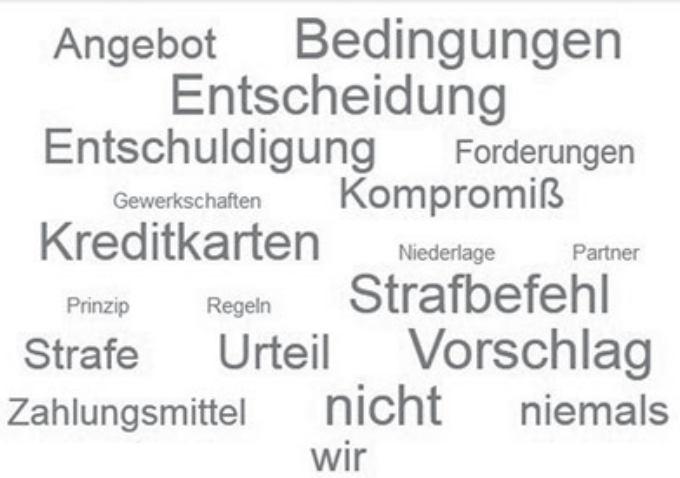

Abb. 10: Schlagwortwolke, Screen-Ausschnitt (DWDS_WP_akzeptieren; 31.05.2018).

Neben dieser Darstellungsform kann das Wortprofil auch in einer klassischen Tabellenform dargestellt werden, vgl. Abb. 11. Neben den statistisch signifikanten Wortformen in Spalte 2 werden in Spalte 3 das auf dem Dice-Koeffizienten basierende Assoziationsmaß logDice und in Spalte 4 die reine Frequenz angegeben. Mithilfe dieser Maße können Kookkurrenzpaare nach Verbindungsstärke bzw. Anziehungskraft sortiert werden.

Über einen Schieber lässt sich einstellen, wie viele Partnerwörter in der Wolken- bzw. Tabellenansicht angezeigt werden sollen (maximal 100 Kookkurrenzpartner ${ }^{125}$ ). Die einzelnen Partnerwörter sind mit den der jeweiligen Analyse zugrundeliegenden Korpusbelegen ${ }^{126}$ verknüpft. Hierdurch ermöglicht das Wortprofil „einen Überblick über den Verwendungszeitraum und die semantischen und pragmatischen Kontexte [...], in denen die syntaktische Relation verwendet wird“ (Geyken 2011a: 16), vgl. Abb. 12.

125 Extrahiert werden können deutlich mehr Kookkurrenzpartner - allerdings ohne die der Auswahl und Zuordnung zugrundeliegenden Belege.

126 Teile der Korpora sind urheberrechtlich geschützt. Es gibt daher Belege, die nicht angezeigt werden dürfen. Andere stehen nur registrierten und angemeldeten Benutzern zur Verfügung. 


\begin{tabular}{|c|c|c|c|c|c|c|c|}
\hline \multicolumn{6}{|c|}{ Lemma } & \multirow{2}{*}{\multicolumn{2}{|c|}{$\begin{array}{c}\text { optional: Wortver } \\
\text { Unterschied } €\end{array}$}} \\
\hline \multicolumn{6}{|c|}{ akzeptieren } & & \\
\hline \multicolumn{2}{|c|}{ Wortart } & \multicolumn{2}{|c|}{ min. $\log$ Dice } & \multicolumn{2}{|c|}{$\min$. Frequenz } & Sortierun & \\
\hline Verb & $\checkmark$ & 0 & $\leqslant$ & 5 & $\forall$ & logDice & $\checkmark$ \\
\hline
\end{tabular}

\begin{tabular}{|c|c|c|}
\hline Überblick & $\log$ Dice $\downarrow_{1}^{\mathrm{q}}$ & Freq. \i $^{9}$ \\
\hline 1. Entscheidung & 5.9 & $1624^{\wedge}$ \\
\hline 2. Bedingungen & 5.9 & 776 \\
\hline 3. Vorschlag & 5.8 & 873 \\
\hline 4. nicht & 5.8 & 11354 \\
\hline 5. Strafbefehl & 5.8 & 314 \\
\hline 6. Kreditkarten & 5.7 & 298 \\
\hline 7. Urteil & 5.5 & 645 \\
\hline 8. Entschuldigung & 5.5 & 276 \\
\hline 9. niemals & 5.3 & 265 \\
\hline 10. wir & 5.2 & 4711 \\
\hline
\end{tabular}

Abb. 11: Tabellarische Übersicht, Screen-Ausschnitt (DWDS_WP_akzeptieren; 31.05.2018).

1: Die Zex, 15.07.2013 (online)

Die Entscheidung der Jury müsse in einem Rechtsstaat aber akzeptiert werden.

2: Die Zex, 14.03.2013 (online)

Und für die Trainer ist es manchmal schwer, die Entscheidungen zu akzeptieren.

3: Die Zet, 28.12.2012, Nr. 52

Meine drei Schwestern und meine Mutter wussten davon und akzeptierten meine Entscheidung, mein Vater hingegen verstie $B$ mich; für ihn bin ich nicht mehr sein Sohn.

4: Die Zes, 24.09 .2012 (online)

Er sei "enttäuscht, einen Spieler zu verlieren, der so viel internationale Erfahrung und so außergewöhnliche Fähigkeiten hat", akzeptiere die Entscheidung nur "widerstrebend".

5: Die Zet, 06.09.2012, Nr. 36

Das heißt nicht, dass politische Entscheidungen einer israelischen Regierung unkritisch akzeptiert werden müssen.

6: Die Zeit, 26.072012, Nr. 30

Er sagt ihn, weil er nicht verstehen kann, warum die anderen seine Entscheidungen nicht akzeptieren kônnen.

7: Die Zet, 23.06.2012 (online)

Er akzeptiere jedoch die Entscheidung des Parlaments, obwohl sein Verteidigungsrecht nicht beachtet worden sei.

8: Die Zea, 28.11 .2011 (online)

Ich akzeptiere die Entscheidung und werde für meine Tat geradestehen ", sagte er.

Abb. 12: Einzelbelege zu Entscheidung akzeptieren, Screen-Ausschnitt (DWDS_WP_akzeptieren; 31.05.2018). 
Auch bei den im Wortprofil abgebildeten Kookkurrenzpartnern handelt es sich überwiegend um automatisch ermittelte Partnerwörter, die - will man Aussagen zu möglichen Lesarten des jeweiligen Suchwortes machen - interpretiert und systematisiert werden müssen:

Wie zuvor beschrieben, ist für die Lesartenunterscheidung des Verbs akzeptieren vor allem die Besetzung der Objektvalenzstelle von besonderem Interesse. Anders als die bisher genannten Abfragesysteme erlaubt das DWDS-Wortprofil eine gezielte Suche hiernach - es ergeben sich nach Extraktion 1.058 Objekt-VerbKookkurrenzen ${ }^{127}$ für akzeptieren. Bei Sichtung der einzelnen Partnerwörter zeigt sich, dass das akzeptieren-Profil des DWDS trotz unterschiedlicher Korpora und Berechnungsgrundlagen mit dem von COSMAS-II ermittelten Profil vergleichbar ist. Auch beim DWDS zeichnen sich die Ausdrücke, die die Objektvalenzstelle des Verbs besetzen können, durch eine große Heterogenität aus. Sie lassen sich, wie in den vorangegangenen Darstellungen gezeigt, folgendermaßen systematisieren: Kookkurrenzpartner, die

- eine Art Angebot beschreiben

Vorschlag, Angebot, Friedensplan, Offerte

- eine Art Zustand beschreiben

Entscheidung, Urteil, Ergebnis, Tod, Schicksal

- ein Zahlungsmittel beschreiben

Kreditkarten, Schecks, Münzen, Bargeld

- als verschieden (z. B. in Bezug auf persönliche Meinungen, soziale Normen) anerkannt werden können

Meinung, Standpunkt, Homosexualität, Vielfalt, Einstellung

- die als höherrangig (z. B. in Bezug auf die eigene Person) anerkannt werden können

Autorität, Verfassung, Demokratie, Werte, Normen

Die Übereinstimmungen beider Profile sprechen für eine gewisse Möglichkeit der Verallgemeinerung der Ergebnisse, insbesondere hinsichtlich der Lesartenbildungen z. B. für das Verb akzeptieren. Sie zeigen auch, dass trotz des deutlich geringeren Korpusumfangs das DWDS-Wortprofil $\mathrm{zu}$ verwertbaren Ergebnissen führt. Es wird daher ergänzend zu dem vom IDS bereitgestellten Verfahren zur Kookkurrenzanalyse in die nachfolgenden Untersuchungen einbezogen.

127 Bei Sichtung der Belege lassen sich einzelne Fehlklassifikationen erkennen: So wurde etwa der Kookkurrenzpartner Gewerkschaften vom Syntaxparser fälschlicherweise als Objekt klassifiziert; in den Belegen besetzt es jedoch ausnahmslos die Subjektposition. Da es sich hierbei jedoch um Einzelfälle handelt, die bei der Beurteilung keinerlei Auswirkungen zeigen, bleiben sie hier unberücksichtigt. 


\subsubsection{Analyse rollenspezifischer Forderungen}

Verben drücken Beziehungen zwischen Individuen im weitesten Sinne (z. B. Personen, Dinge oder Orte) aus (Peter öffnet die Konservendose) oder sie sagen etwas über einzelne Individuen aus (Peter hält an). Damit eröffnen Verben mit ihren Bedeutungen

bestimmte Stellen für die Angabe von Personen, Dingen oder Umständen, die im bezeichneten Sachverhalt eine bedeutungskonstitutive Rolle spielen: wer oder was tätig ist und etwas bewirkt, wer oder was betroffen oder verändert wird, wem was geschieht, wo was geschieht usw. Die semantische Funktion dieser Stellen nennt man semantische Rolle (auch: thematische Rolle, Tiefenkasus oder Theta-Rolle).

(Primus 2015: 79)

Die in den Verblesarten semantisch festgelegten ein- bzw. mehrstelligen Relationen spielen eine zentrale Rolle bei metasprachlichen, d.h. sprachreflexiven Aufgaben $^{128}$ (vgl. Primus 2012, 2015) und damit auch bei der hier angestrebten Lesartendifferenzierung. Schauen wir uns zur Verdeutlichung dieser Überlegungen noch mal die in (90) dargestellte und in (107) wiederholte Bedeutungsparaphrase für das Verb öffnen (in der dort dargestellten Lesart) an:

(107) öffnen

i.S.v. jemand bewirkt, dass etwas zugänglich ist; aufmachen

Die hier verwendete Bedeutungsumschreibung erklärt „die Verbbedeutung nicht direkt, sondern mit Hilfe der Verursacherrolle („bewirken, dass“) und der Rolle der Sache, die eine Veränderung erfährt (,dass etwas offen ist/wird“, d. h. was sich

128 Darüber hinaus spielen semantische Rollen auch beim Erwerb von Verbbedeutungen eine entscheidende Rolle (vgl. Primus 2015: 80ff.). Wenn Kinder z. B. das Wort geben kennenlernen, dann handelt es sich dabei anfänglich allein um eine lautliche Geste, ,die zunächst nur die geteilte Aufmerksamkeit auf ein ganzheitlich erfahrenes, kulturell relevantes Interaktionsmoment in der unmittelbar gemeinsam erlebten wie prozedural durchlebten Situation lenkt" (Konerding 2015: 68). Daran anschließend lernen die Kinder „die Mitspielerrollen, die den Akt des Gebens stets begleiten: der Gebende, der gegebene Gegenstand und die Person, der etwas gegeben wird; tatsächlich können wir uns nicht einmal einen Akt des Gebens ohne diese Mitspielerrollen vorstellen.“ Tomasello (2006: 173)

Darstellungen wie diese zeigen, was Kinder u. a. beim Erwerb von Verben zu leisten haben: Sie versuchen permanent, aus lautlichen Gesten durch Klassifikationen und Verallgemeinerungen abstrakte Sprachkonstruktionen abzuleiten (vgl. de la Rosa 2012). Da Verben Beziehungen zwischen Personen und Sachen ausdrücken und diese Beziehungen semantisch festgelegt sind, geht somit „der Erwerb eines Verbs mit dem Erwerb seiner semantischen Rollen“ (Primus 2015: 80) einher. 
verändert)“ (Primus 2015: 81). Auch die hier benutzten Wörterbücher greifen - mit Ausnahme des DWDS ${ }^{129}$ - auf eine solche Darstellungsweise zurück:

(108) a. DUDEN $2012^{4}$ _öffnen

„bewirken, dass etwas offen ist“

b. E-VALBU_öffnen; 31.05.2018

„jemand/etwas bewirkt durch Lösen einer Verschlussvorrichtung, dass etwas offen oder zugänglich ist, benutzt werden kann; aufmachen“

Der Vorteil derartiger Bedeutungsumschreibungen liegt darin, dass sie - anders als Bedeutungsparaphrasen, die die Satzglied-Bedeutung mit Hilfe sortaler Merkmalsforderungen in Isolation beschreiben (vgl. Helbig/Schenkel 1983 $)^{130}$-, auch „die semantische Funktion, die sie relativ zum Verb übernehmen, d.h. deren semantische Rolle, angeben“ (Primus 2015: 81). Sie erlauben damit eine Festlegung der Weise, wie das Relat in die von der jeweiligen Verblesart beschriebenen Situation involviert ist (vgl. Jacobs 2003: 389). Die Subjekt-Relatposition von öffnen fordert z. B. in der hier dargestellten Lesart „,von der entsprechenden Entität nicht nur Belebtheit, sondern unter anderem auch, dass [...] [sie, TvdB] die beschriebene Situation kontrolliert, d.h. aus eigenem Antrieb initiiert und ihren Verlauf beeinflusst“ (ebd.).

Da sich traditionelle Rollen wie Agens, Patiens oder Rezipient als weitgehend unbrauchbar erwiesen haben (vgl. Primus 2012a), hat sich ausgehend von Lakoff (1977) und Dowty (1991) die Idee eines mehrdimensionalen, auf Ähnlichkeitsbeziehungen basierenden Proto-Rollen-Ansatzes entwickelt. Die dabei angenommenen Proto-Rollen-Dimensionen ermöglichen es, spezifischere semantische Rollen zu definieren und z. B. als agens- oder patiensähnlich auszuweisen (vgl. Primus 2012a). In der Forschungsliteratur sind unterschiedliche Ausprägungen dieser Idee $\mathrm{zu}$ finden. Allen Vorschlägen gemeinsam ist jedoch, dass auch nach der

129 Im DWDS wird öffnen umschrieben als etw. Zugeschlossenes aufschließen. Dabei wird auf die Angabe semantischer Merkmale und/oder semantischer Rollen gänzlich verzichtet.

130 Wie die Darstellung in (VI) zeigt, beschreiben Helbig/Schenkel (19837) in ihrem Wörterbuch zur Valenz und Distribution deutscher Verben ausschließlich sortale Merkmalsforderungen im o. g. Sinne (vgl. Helbig/Schenkel (19837:318)):

(VI) öffnen (aufmachen)

Satzglied Nominativ (Sn) $\rightarrow$ menschliches Wesen (Hum)

Satzglied Akkusativ (Sa) $\rightarrow$ unbelebtes Wesen (-Anim)

Das Wörterbuch wurde 1968 erstmals veröffentlicht und noch vor den frühen Arbeiten zu semantischen Rollen (vgl. z. B. Fillmores (1968)) konzipiert (vgl. Primus 2015). 
Wende $\mathrm{zu}$ einer flexibleren Analyse ${ }^{131}$ die Frage bislang noch immer ungeklärt ist, mit welchem Inventar konkreter Rollenforderungen (wie Kontrolle, Verursachung, Wahrnehmung) die zu beschreibenden Zusammenhänge, z. B. zwischen semantischer und kategorialer Valenz, am besten erfasst werden können.

Vor diesem Hintergrund und weil man für die hier durchzuführende Lesartendifferenzierung m. E. keine ausgeklügelte Rollenklassifikation benötigt, wird im Folgenden in Anlehnung an Primus (2015) versucht, rollenspezifische Forderungen anzugeben mit Hilfe von verblexemspezifischen Angaben und ggf. einiger sehr allgemeiner rollensemantischer Begriffe wie Verursachung, Veränderung, Besitz oder Ort. So kommen viele

einflussreiche Ansätze [...] mit verblexemspezifischen Rollen aus (Tomasello 1992, Sag/ Wasow 1999). Allgemeinere rollensemantische Begriffe wie Verursachung und Veränderung dienen dazu, verblexemspezifische Rollen zu Klassen zusammenzufassen. Diesen Klassen entsprechen die traditionellen semantischen Rollen wie Agens und Patiens.

(Primus 2015: 101)

Eine solche Vorgehensweise erscheint wegen der hier zugrundegelegten Wörterbücher, die auf eine ähnliche Strategie zurückgreifen, sinnvoll und lohnenswert. Die Autoren von E-VALBU geben z. B. an, dass in den jeweiligen Wörterbuchartikeln unter dem Schlagwart im Sinne von die Bedeutung der jeweiligen Lesart angegeben wird ,und zwar so, dass man die inhaltliche Funktion (semantische Rolle) der Komplemente in einem Satz mit dem Verb in dieser Bedeutung erkennen kann“ (E-VALBU; cf. Benutzerhinweise), ohne dabei auf die traditionellen Rollenbegriffe wie Agens, Patiens oder Rezipient zurückzugreifen.

Ermittelt werden die zu spezifizierenden Stellen mit Hilfe der in 4.2.1 dargestellten Bedeutungsparaphrasen. In diesen enthalten sind die für die jeweilige Verblesart bedeutungskonstitutiven Stellen. Ein Verb wie geben in einem Satz wie Peter gibt Susi das Buch könnte z. B. folgendermaßen paraphrasiert werden:

(109) geben

i.S.v. jemand hält jemandem/etwas etwas hin, damit er es erreichen kann; reichen (vgl. E-VALBU_geben; 31.05.2018)

Die Bedeutungsparaphrase eröffnet drei bedeutungskonstitutive Stellen. Dabei handelt es sich um von der hier dargestellten geben-Lesart präsupponierte Mit-

131 Darüber hinaus kann man leicht erkennen, dass auch vor dem Hintergrund eines ProtoRollen-Ansatzes ,die Zahl der traditionellen Rollen sehr schnell anwächst und die erhoffte Überschaubarkeit des Rollensystems zusammenbricht“ (Primus 2015: 101). 
spieler (vgl. Heringer 1984: 49), die mit Hilfe verblexemspezifischer Rollen als Gebender, Empfänger und überreichter Gegenstand bezeichnet werden können. Allgemeinere rollensemantische Begriffe können anschließend dazu genutzt werden, verblexemspezifische Rollen zu Klassen - den traditionellen semantischen Rollen - zusammenzufassen. Mit Hilfe der Verursacher-Relation kann z. B. der erste Mitspieler, der Gebende, als Agens klassifiziert werden. Dagegen kann mit Hilfe der Empfänger-Relation der zweite Mitspieler, der Empfänger, als Rezipient bezeichnet werden. Der dritte Mitspieler, der überreichte Gegenstand, kann schließlich mittels der Betroffenheits-Relation als Patiens eingeordnet werden.

\subsection{Fragebogenuntersuchung}

Die in Kapitel 5 folgenden Untersuchungen zu den semantischen Spezialisierungen impliziter Argumente werden zeigen, dass mit den zuvor vorgestellten Methoden zur Polysemiedifferenzierung nicht immer hinreichende Aussagen zum Lesartenspektrum der zu untersuchenden Verben gemacht werden können. Daher wurde ergänzend ein Fragebogen als

eine mehr oder weniger standardisierte Zusammenstellung von Fragen, die Personen zur Beantwortung vorgelegt werden mit dem Ziel, deren Antworten zur Überprüfung der den Fragen zugrundeliegenden theoretischen Konzepte und Zusammenhänge zu verwenden.

(Porst 1996: 738)

entwickelt.

Ziel des hier eingesetzten Fragebogens ${ }^{132}$ (s. Fragebogen - Teil 2) ist, einzelne, mit Hilfe anderer Ressourcen gewonnener Bedeutungshypothesen ausgewählter Verben zu überprüfen. Dazu wurden verschiedene Informantengruppen gebeten, die Bedeutungen einzelner Testsätze möglichst genau zu umschreiben. Zur Beurteilung der Testsätze standen ihnen unterschiedliche, auf Korpusrecherchen basierende geschlossene Antwortmöglichkeiten zur Verfügung, vgl. (Abb.13). Bei Unsicherheit waren die Befragten angehalten, sich entsprechend ihrer Tendenz zu entscheiden:

Dass das geschlossene Antwortformat nur begrenzte und definierte Antwortkategorien vorgibt, die Probanden folglich nur eine Auswahl aus einem eingeschränkten Bereich treffen können (vgl. Betz 2016: 101), wird vor dem Hintergrund der Ergebnisse eines Pretests in Kauf genommen. So ergab dieser, dass die Befragten bei einer offenen Fragestellung, bei der keine Antwortkate-

132 Der vollständige Fragebogen ist in Kapitel 8 abgebildet; ausführliche Angaben zur Konzeption und Durchführung finden sich in Kapitel 1.3. 
Fridolin und seine Frau Theresa haben den Tag im Garten verbracht. Als sie gemeinsam reingehen, fragt er sie: „Schatz, wollen wir gleich noch ins Kino?" Sie antwortet: "Oh ja, dann bade ich mich jetzt. Danach können wir los."
Theresa wäscht sich in der Badewanne

Der Vorgang ist eher

$\begin{array}{lll}\square \text { aktiv } & \text { oder } \square \text { inaktiv } \\ \square \text { kürzer } & \text { oder } \square \text { länger }\end{array}$

Theresa liegt von Wasser bedeckt in der Badewanne Der Vorgang ist eher

$\square$ aktiv oder $\square$ inaktiv

Abb. 13: Beispiel Fragebogen (Teil 2).

gorien vorgegeben waren, häufig die beobachtbaren Bedeutungsunterschiede zwar wahrnahmen - so gaben etwa 90\% der im Pretest Befragten an, dass Sätze wie Peter badet und Peter badet sich für sie nicht gleichbedeutend sind -, diese jedoch nicht oder nur unzureichend beschreiben konnten. Um dennoch (zeitökonomisch) auswertbare Daten zu erhalten, wurden ausgehend von umfangreichen Korpusrecherchen (vgl. hierzu die Darstellungen der Einzelverben) geschlossene Antwortformate formuliert.

Mit der hier gewählten Vorgehensweise wird auch versucht, der allgemein beobachtbaren Tendenz, dass Probanden aufgrund des höheren Zeitaufwandes bei offenen bzw. halboffenen Aufgabenformaten geschlossene Itemformate häufig vorziehen (vgl. Raab-Steiner/Benesch 20123), zu entsprechen. Zudem zeichnen sich geschlossene Items durch eine höhere Objektivität sowie eine bessere Auswertbarkeit der Daten aus (vgl. ebd.).

\subsection{Bewertung}

Zusammenfassend lässt sich festhalten, dass keine der vorangehend beschriebenen Methoden zur Lesartendifferenzierung für sich genommen eine zufriedenstellende Lesartenunterscheidung ermöglicht. Wörterbuchanalysen, Angaben von Paraphrasen und Synonymen, Untersuchungen syntaktischer Eigenschaften, Kookkurrenz- und Kolligationsanalysen, Analysen rollenspezifischer Merkmale sowie Fragebogenuntersuchungen geben zwar gute Hinweise auf das Vorliegen unterschiedlicher Verwendungsweisen; jede Methode für sich ist jedoch auch mit z. T. erheblichen Problemen verbunden.

Daher wird im Rahmen dieser Arbeit nicht lediglich ein Verfahren zur Lesartendisambiguierung eingesetzt; vielmehr wird auf eine Art mehrgliedrige Verfahrensweise zurückgegriffen. Dabei werden die Beschreibungen der hier ausgewählten Verben in den drei großen (Valenz-)Wörterbüchern der Gegenwartssprache DUDEN, DWDS und E-VALBU zugrundegelegt. Bei Sichtung entsprechender Wörterbucheinträge zeigt sich, dass die drei Wörterbücher sowohl 
in der Anzahl der angenommenen Lesarten als auch bei deren Zusammenfassung zu einzelnen Unterlesarten z. T. erheblich voneinander abweichen (vgl. z. B. die Einträge zum Verb spielen). Zudem entsprechen die Wörterbucheinträge nicht immer einem allgemein intuitiven Verständnis (vgl. die Einträge zum Verb akzeptieren). Dies ist besonders problematisch, da die in den Wörterbüchern zugrundegelegten Kriterien zur Lesartenunterscheidung nicht offengelegt und auch in einem nachgelagerten Analyseschritt häufig nicht erkennbar werden.

Daher werden zusätzlich die zuvor dargestellten traditionellen Verfahren zur Abgrenzung von Verwendungsweisen herangezogen. Dabei kommt der Angabe von semantischen Bedeutungsparaphrasen und Synonymen eine besondere Bedeutung zu, werden in ihnen ,in abstrahierter Form die verschiedensten Teilinformationen zur Bedeutung und Verwendung eines Stichworts gebündelt“ (Storjohann 2005: 182). Damit sind in Bedeutungsparaphrasen gleich zwei wichtige Informationsarten enthalten: Zum einen Informationen zur Bedeutung des $\mathrm{zu}$ beschreibenden Wortes, und zum anderen Informationen $\mathrm{zu}$ Beziehungen $\mathrm{zu}$ anderen Wörtern, die das jeweilige Wort in der vorliegenden Bedeutung aufweist (vgl. ebd. 183f.).

Letztere geben Auskunft über die bedeutungskonstitutiven Leerstellen einer Verblesart, die mit lesartenspezifischen Forderungen und Restriktionen verbunden sind. Ermitteln lassen sich die kategorialen Valenzforderungen vor allem mit Untersuchungen syntaktischer Eigenschaften. Im Zusammenhang mit semantischen Valenzforderungen kommen Verfahren wie die Kollokations- bzw. Kolligationsanalyse zur Ermittlung sortal spezifischer Forderungen und Restriktionen sowie Verfahren zur Überprüfung rollenspezifischer Forderungen zum Einsatz. Insbesondere dort, wo die zuvor genannten Verfahren $\mathrm{zu}$ nicht eindeutigen Ergebnissen führen, werden zur Überprüfung einzelner Bedeutungshypothesen ergänzend Fragebogenuntersuchungen durchgeführt.

Ein solcher Methodenmix ermöglicht eine möglichst feingliedrige Erfassung der Verblesarten. Welche der genannten Kriterien bei den $\mathrm{zu}$ untersuchenden Verben im Einzelnen berücksichtigt werden und welches Gewicht ihnen bei den Lesartenunterscheidungen beigemessen wird, hängt aufgrund der Spezifika des Untersuchungsgegenstandes von den zu untersuchenden Verben ab und wird daher einzelverbbezogen entschieden (vgl. Kapitel 5).

Im Anschluss folgen nun umfangreiche Untersuchungen $\mathrm{zu}$ den semantischen Spezialisierungen impliziter Argumente. Dabei geht es ausgehend von den in F1 und F2 formulierten Forschungsfragen vor allem darum zu klären, inwieweit es sich bei den in der Literatur beschriebenen - und im Sinne von F1 ggf. zu ergänzenden - semantischen Effekten um Folgen der Weglassung handelt, oder ob im Sinne von F2 bereits vor Argumentweglassung bestehende Lesartendifferenzierungen vorhanden sind. Zur Beantwortung dieser Fragen werden die ein- 
zelnen Verwendungsweisen der Verben soweit ermittelt und unterschieden, wie es zur Beantwortung eben dieser Fragestellungen notwendig ist. Metonymische und metaphorische Prozesse von Lesartenerweiterungen sowie ihre Auswirkungen auf Weglassungsoptionen bleiben dabei unberücksichtigt. Ein Anspruch auf Vollständigkeit wird nicht erhoben.

Dabei gilt bei den zur Lesartenunterscheidung angegebenen Bedeutungsbeschreibungen, „was für andere Beschreibungen auch gilt: sie sind Beschreibungen zu einem bestimmten Zweck, sie sind Beschreibungen unter bestimmten Aspekten, und es gibt keine Vollständigkeit der Beschreibung“ (Gloning 1996: 204). Daher wird es im Folgenden bei der Darstellung der unterschiedenen Verwendungsweisen immer wieder auch zu Vereinfachungen - vgl. z. B. die Nicht-Berücksichtigung des Situationsrelats [s] (s. Anmerkungen in Kapitel 2) - kommen. Um dennoch aussagekräftige Ergebnisse zu erzielen, wird bei allen Lesartenspezifikationen von folgender Normalvorgabe ausgegangen: „Wir interpretieren sprachliche Ausdrücke nicht metaphorisch, gehen von einer ernsthaften, rationalen Kommunikation aus, und halten Referenten, Orte und Zeitpunkte konstant.“(Primus 2012a: 10). 